\title{
A multifunctional-targeted nanoagent for dual-mode image-guided therapeutic effects on ovarian cancer cells
}

This article was published in the following Dove Medical Press journal: International Journal of Nanomedicine

\section{Chunyan Chen' \\ Jiangchuan Sun' \\ Shuning Chen' \\ Yujiao Liu' \\ Shenyin Zhu ${ }^{2}$ \\ Zhigang Wang ${ }^{3}$ \\ Shufang Chang'}

'Department of Obstetrics and Gynecology, The Second Affiliated

Hospital of Chongqing Medical University, Chongqing 4000 I0, China;

${ }^{2}$ Department of Pharmacy, The First

Affiliated Hospital of Chongqing

Medical University, Chongqing

4000 I6, China; ${ }^{3}$ Chongqing Key

Laboratory of Ultrasound Molecular

Imaging, Institute of Ultrasound

Imaging, Second Affiliated Hospital

of Chongqing Medical University,

Chongqing 400010 , China
Correspondence: Shufang Chang

Department of Obstetrics and

Gynecology, The Second Affiliated

Hospital of Chongqing Medical University,

74 Linjiand Road, Yuzhong District,

Chongqing 4000 I6, China

Tel +8602363693279

Fax +86023 65I0 4238

Email shfch2005@163.com
Purpose: Nanomedicine has emerged as a novel therapeutic modality for cancer treatment and diagnosis. Lipid-polymer hybrid nanoparticles (LPHNPs) are core-shell nanoparticle (NP) structures comprising polymer cores and lipid shells, which exhibit complementary characteristics of both polymeric NPs and liposomes. However, it is difficult to wrap perfluoropentane (PFP) into core-shell NPs in the existing preparation process, which limits its application in the integration of diagnosis and treatment.

Methods: The folate-targeted LPHNPs-loaded indocyanine green/PFP-carrying oxygen (TOI_HNPs) using a combination of two-step method and solution evaporation technique for the first time. The essential properties and dual-mode imaging characteristics of developed NPs were determined. The cellular uptake of TOI_HNPs was detected by confocal microscopy and flow cytometry. The SKOV3 cell viability and apoptosis rate were evaluated by 3-(4,5-dimethyl2-thiazolyl)-2,5-diphenyl-2-H-tetrazolium bromide (MTT) assay and flow cytometry. The ROS was demonstrated by fluorescence microplate reader and the expression of hypoxia-inducible factor 1-alpha (HIF-1 $\alpha$ ) and IL-6 was detected by Western blot.

Results: TOI_HNPs showed spherical morphology with particle size about (166.83 \pm 5.54$) \mathrm{nm}$ and zeta potential at $-(30.57 \pm 1.36) \mathrm{mV}$. It exhibited better stability than lipid NPs and higher encapsulation efficiency as well as active targeting ability than poly (lactic-co-glycolic acid) (PLGA) NPs. In addition, the novel NPs could also act as the contrast agents for ultrasound and photoacoustic imaging, providing precision guidance and monitoring. Furthermore, TOI_HNPsmediated photo-sonodynamic therapy (PSDT) caused more serious cell damage and more obvious cell apoptosis, compared with other groups. The PSDT mediated by TOI_HNPs induced generation of intracellular ROS and downregulated the expression of HIF-1 $\alpha$ and IL-6 in SKOV3 cells Conclusion: This kind of multifunctional-targeted nanoagent may provide an ideal strategy for combination of high therapeutic efficacy and dual-mode imaging guidance.

Keywords: core-shell nanoparticle, phase transformation, photoacoustic imaging, laser, ultasound, photo-sonodynamic therapy

\section{Introduction}

Ovarian cancer is one of the most serious diseases which is severely threatening the female population. There were about 14.1 million new cancer cases and 8.2 million deaths that occurred in 2012 worldwide. ${ }^{1}$ Difficulties in early diagnosis and unsatisfactory treatment lead to increasing mortality. Surgery has little effect on micrometastases, and nonspecific chemotherapy and radiotherapy often causes systemic side effects. ${ }^{2}$ Therefore, it is necessary for improved technology on efficient ovarian cancer diagnosis 
and treatment. The rapid development of nanotechnology in the nanomedical products has brought great promise for cancer treatment strategies. ${ }^{3-5}$

In the past few decades, intensive researches have been made in nanomedicine and several nanoplatforms have been approved for clinical trials. ${ }^{3,6}$ Nanoplatform has the following characteristics: 1) optimal size: nanosized particles achieved passive targeting through enhanced permeability and retention effect; ${ }^{7}$ 2) drug loading: nanocarriers can encapsulate some molecules such as hydrophobic drugs, contrast agents, and siRNA for the high surface-to-volume ratio; ${ }^{3}$ 3 ) easily modifiable: nanoparticles (NPs) are modified by specific ligands for biocompatibility or active targeting; ${ }^{8}$ and 4) favorable physicochemical characteristics: imaging and treatment are carried out using the unique properties of the nanocarrier itself (such as semiconductor quantum dots) ${ }^{9}$ or encapsulating molecules (such as photosensitizers). ${ }^{10}$ Hence, multifunctional NPs provide an opportunity for precise imaging and targeted therapy.

Among all reported NP systems, polymers and lipids have been commonly used as two major drug nanocarriers. ${ }^{11,12}$ Generally, polymeric NPs exhibit better stability during storage, high structural integrity, and controlled release capability, so they are widely used. The polymer poly (lacticco-glycolic acid) (PLGA) is approved by the U.S. Food and Drug Administration (FDA) and applied in biomedical field. However, the low biocompatibility and poor modification of PLGA NPs shortened their long circulation time and limited their targeted aggregation. ${ }^{13}$ Compared with polymer NPs, lipid NPs have been considered as more ideal drug delivery because of their distinguishing feature: amphiphilicity, long circulation time, excellent biological compatibility, and readily functionalized. Nevertheless, the main drawback of lipid NPs is instability, resulting in structural damage and fast drug release. ${ }^{14}$ Therefore, novel systems known as lipid-polymer hybrid nanoparticles (LPHNPs) that are core-shell NP structures comprising polymer cores and lipid shells have been introduced to combine these advantages and overcome the shortcomings of the two nanocarriers. ${ }^{11,12}$

LPHNP systems were described as a lipid layer that covers the surface of the polymeric core. The space between polymeric core and lipid layer is usually occupied by chemotherapy drugs and functional molecular probes. Indocyanine green (ICG) has been proved to be an ideal sensitizer for photodynamic therapy (PDT) or sonodynamic therapy (SDT) by production of ROS. ${ }^{7,8,15}$ Perfluorocarbons (PFCs) are great oxygen carriers that improve the oxygen concentration at the target site. ${ }^{16}$ Specially designed PFC NPs can convert into microbubbles under the action of laser irradiation by incorporating optical absorbing materials into nanocarriers (termed as optical droplet vaporization [ODV]). With exposure of the near-infrared (NIR) laser, the optical absorbers could appear as transient thermoelastic expansion to generated conventional photoacoustic (PA) signals. ${ }^{17}$ Furthermore, once PFC droplets have been triggered into gas phase, it can also provide excellent contrast for ultrasound (US) imaging. ${ }^{17-19}$ The folate receptor (FR) is a potential biological target, which could be overexpressed on the surface of ovarian cancer cells, whereas low to negligible levels are expressed in normal tissues. ${ }^{20}$ We propose to synthesize targeted hybrid NPs, which modified folic acid (FA) on the lipid shell and encapsulating ICG and PFC-carrying oxygen in the PLGA core for dual-mode imaging and ovarian cancer treatment. However, it is difficult to wrap perfluoropentane (PFP, one of fluorocarbon with low boiling point) into core-shell NPs in the existing preparation process as the contradiction of lipid needs heating, but the PFP cannot be heated during synthesis, ${ }^{11,12,21-24}$ which limits LPHNPs application in the integration of diagnosis and treatment.

In this study, we successfully constructed one kind of folate-targeted LPHNPs-loaded ICG/PFP-carrying oxygen (TOI_HNPs) systems by combining two-step method and solution evaporation technique for the first time. It exhibited better stability than lipid NPs, as well as higher encapsulation efficiency and active targeting ability than PLGA-NPs. Upon the proper laser energy irradiation, large amounts of microbubbles can be generated through the PFP phasetransition process, providing excellent contrast for both PA and US imaging. Furthermore, ICG as a sensitizer could induce ROS generation by photo-sonodynamic therapy (PSDT) to achieve a certain therapeutic effect. This kind of multifunctional-targeted nanoagent may provide an ideal strategy for combination of high therapeutic efficacy and dual-mode imaging guidance.

\section{Materials and methods Materials}

ICG was purchased from Sigma-Aldrich Co. (St Louis, MO, USA). PLGA (lactide: glycolide $=50: 50$, molecular weight $=12,000 \mathrm{Da}$ ) was obtained from the Shan-dong Key Laboratory of Medical Polymer Materials (Shandong, China). Phosphatidylcholine (DPPC), 1,2-dipalmitoyl-sn-glycero-3phospho-(1'-rac-glycerol) (DPPG), 1,2-distearoyl-sn-glycero3-phosphoethanolamine- $N$-[folate(polyethyleneglycol)-2000] (DSPE-PEG (2000)-FA), and cholesterol were purchased from Avanti Polar Lipids Inc. (Alabaster, AL, USA). PFP was obtained from Fluka (St Louis, MO, USA). Polyvinyl alcohol (PVA, 87\%-90\%) was also obtained from Sigma-Aldrich Co. 
Singlet Oxygen Sensor Green (SOSG) was purchased from Thermo Fisher Scientific (Waltham, MA, USA). Antibodies against hypoxia-inducible factor 1-alpha (HIF-1 $\alpha$ polyclonal, 1:500, NB100-479) were purchased from Novus Biologicals (Littleton, CO, USA). IL-6 polyclonal (1:1,000, DF6087) was purchased from Affinity Biosciences, Inc. (Cincinnati, OH, USA). The fluorescence dye, 1,1'-dioctadecyl3,3,3',3'-tetramethylindocarbocyanine perchlorate (DiI) was purchased from Sigma-Aldrich Co. Hoechst 33342/propidium iodide double-staining kit was obtained from Nanjing Jiancheng Bioengineering Institute (Nanjing, China). FA, 2-(4-amidinophenyl)-6-indolecarbamidine dihydrochloride (DAPI), 3-(4,5-dimethylthiazol-2-yl)-2,5-diphenyltetrazoliumbromide (MTT), 2',7'-dichlorofluorescin diacetate (DCFH-DA) were all supplied by Beyotime Biotechnology (Shanghai, China). All other reagents used in this study were commercial products of analytical grade.

\section{Formulation of NPs}

TOI_HNPs were prepared using a combination of the twostep method and the solution evaporation method. First, the lipid mixture of DPPC, DPPG, DSPE-PEG-(2000)-FA, and cholesterol $(5: 2: 2: 1$, molar ratio) was dissolved in trichloromethane-methanol $(4: 1, \mathrm{v} / \mathrm{v})$ and a lipid film in a rotary evaporator (Yarong Inc., Shanghai, China) was formed. The PFP $(200 \mu \mathrm{L})$ was bubbled with oxygen gas till saturation and added to oxygen enriched ICG aqueous solution $(500 \mu \mathrm{L}$, $3 \mathrm{mg} / \mathrm{mL}$ ) to get a uniform mixture by ultrasonic vibration. Then the mixture was bubbled with oxygen again. After that, the PLGA-methylene chloride solution $(2 \mathrm{~mL}, 25 \mathrm{mg} / \mathrm{mL}$ ) was added into the mixture and sonicated by the ultrasonic probe (Sonics \& Materials, Inc. Newtown, CT, USA) to form PLGA NPs emulsion (PFP-carrying oxygen/ICG-loaded PLGA NPs). Subsequently, the preformed lipid film ( $5 \mathrm{~mL}$, $1 \mathrm{mg} / \mathrm{mL}$ ) was added to the suspension for emulsification to obtain lipid shell-PLGA core NPs. Finally, the organic solvent was removed by stirring with a magnetic stirrer (Haimen Kylin-Bell Lab Instruments Co., Ltd. Jiangsu, China) for 4-6 hours until all organic solvents were evaporated. Next, the precipitate obtained by centrifugation (Biofuge Stratos Centrifuge; Thermo Fisher Scientific, Waltham, MA, USA) is resuspended in water and stored at $4{ }^{\circ} \mathrm{C}$ for application. OI_PNPs (PLGA NPs-encapsulated ICG and PFP-carrying oxygen) were prepared by replacing the lipid film with PVA aqueous solution $(5 \mathrm{~mL}, 5 \% \mathrm{w} / \mathrm{v})$, since it is the most common emulsion stabilizer used in the pharmaceutical field to prepare micro and nanosystems. ${ }^{7,25}$ No ICG- or PFP-carrying oxygen is added into TO_HNPs and TI_HNPs, respectively. Other processes are similar to the above steps.
TOI_LNPs (folic-targeted lipid NPs-encapsulated ICG and PFP-carrying oxygen) were synthesized by a single-step emulsion method based on previously published studies. ${ }^{19,26}$ Briefly, the lipid film was prepared by rotary evaporator, then it was resuspended in double-distilled water as the lipid hydration membrane, which was dropped in the PFP and ICG aqueous solution filled with oxygen for 5 minutes under acoustic vibration in an ice bath. Finally, the obtained suspension was centrifuged three times (3,000 rpm, 5 minutes) and the supernatant was discarded and the precipitate was suspended in water for reserve.

\section{Characterization of the NPs}

\section{Primary physicochemical properties}

The morphology of TOI_HNPs was observed by confocal laser scanning microscopy (CLSM, Leica TCS SP5, Heidelberg, Germany) and the structure was characterized by using transmission electron microscope (TEM, FEI Tecnai G2 F20, FEI Company, Hillsboro, OR, USA). The size distribution, zeta potential, and polydispersity index (PDI) of NPs were determined by dynamic light scattering (DLS) using a Malvern Zetasizer Nano ZS (Malvern Instruments, Malvern, UK). The phase-transition ability after being heated by a temperature control heating-plate or irradiated by a NIR laser exciter was observed by inverted fluorescence microscope (Eclipse Ti, Nikon Corporation, Tokyo, Japan). And the size stability of the TOI_LNPs, OI_PNPs, and TOI_HNPs in water was observed after storing at $4^{\circ} \mathrm{C}$ varying from day 0 to day 30. The entrapment efficiency (EE) and the ICG-loading efficiency (LE) were determined in triplicate by an ultraviolet-visible (UV-Vis) spectrophotometer (260-Bio; Thermo Fisher Scientific). Briefly, the supernatant of centrifugation and washing process were collected and the concentration of ICG in the supernatant was obtained from the standard curve of free ICG in the same solvent by absorbance measuring at $780 \mathrm{~nm}$. The EE and the drug LE were calculated using the following equation:

$$
\begin{gathered}
\text { The amount of ICG in formulation }- \\
\text { EE }(\%)=\frac{\text { The amount of ICG in supernatant }}{\text { The amount of ICG in formulation }} \times 100 \% \\
\text { The amount of ICG in formulation }- \\
\text { LE }(\%)=\frac{\text { The amount of ICG in supernatant }}{\text { Total amount of NPs }} \times 100 \% .
\end{gathered}
$$

\section{Optical properties}

The absorption spectra of free ICG, TOI_LNPs, OI_ PNPs, and TOI_HNPs were obtained using an UV-Vis 
spectrophotometer. Fluorescence spectra were obtained using fluorescence spectroscopy (Cray Eclipse; Agilent Technologies, Santa Clara, CA, USA) with excitation at $760 \mathrm{~nm}$ to monitor ICG. The maximum absorbance and fluorescence intensity of NPs at different times were measured to assess the optical stability of ICG in different situations. An equal concentration of $8 \mu \mathrm{g} / \mathrm{mL}$ ICG was used.

\section{In vitro oxygen and ICG release study of NPs}

The $2 \mathrm{~mL}$ liquid paraffin was added to isolate the air. Then, the concentration of dissolved oxygen was measured before and after NIR laser irradiation (808 nm, Nd:YAG laser, $1.5 \mathrm{w} / \mathrm{cm}^{2}, 2$ minutes $)$ and US exposure ( $1 \mathrm{w} / \mathrm{cm}^{2}, 1$ minute) through a portable dissolved oxygen meter (JPBJ-608, Shanghai INESA Scientific Instrument Co. ltd., shanghai, China) by adding the free ICG, TO_HNPs, TI_HNPs, OI_PNPs, TOI_LNPs, and TOI_HNPs (ICG concentration $8 \mu \mathrm{g} / \mathrm{mL}$, NPs) into 24-well plate, respectively. And the same amount degassed water was used as a control group.

The ICG release profiles of OI_PNPs, TOI_LNPs, and TOI_HNPs at PBS $(\mathrm{pH}=7.4)$ and $10 \%$ BSA solution were investigated. The NPs suspension was kept in a metabolic shaking incubator at $37^{\circ} \mathrm{C}$ and shaken at $100 \mathrm{rpm}$. The ICG release was investigated by an UV-Vis spectrophotometer. At a certain time point, three samples from the drug-releasing system were taken out and centrifuged to collect the supernatant. The concentration of ICG in the supernatant was measured with the absorbance. Release kinetic studies were done up to 72 hours.

\section{Dual-mode imaging of TOI_HNPs}

The function of TOI_HNPs as a contrast agent for dual-mode imaging was evaluated in vitro using an agar-gel phantom ( $2 \%$ agar $\mathrm{w} / \mathrm{v}$ in degassed water) followed in the previous studies. ${ }^{17-19,26}$ A linear probe $(5-12 \mathrm{MHz})$ of ultrasonic diagnostic instrument (MyLab 90, Esaote, Italy) was used to investigate the standard $\mathrm{B}$ mode and contrast mode before and after laser irradiation (808 nm, Nd:YAG laser, $1.5 \mathrm{~W} / \mathrm{cm}^{2}$, 2 minutes) in degassed water, free ICG, TO_HNPs, and TOI_ HNPs groups. The echo intensity (EI) was analyzed using an US image analysis software (Model: DFY; Chongqing Medical University, Chongqing, China). Similarly, the PA images and PA intensity variation in different samples were observed by the VEVO LASR PA imaging system (VIVO 2100, FUJIFILM Visual Sonic, Inc., Bothell, WA, USA).

\section{Cell culture and cellular uptake}

Human SKOV3 ovarian cell line was a generous gift from Professor Ronald X. Xu at University of Science and
Technology of China (Hefei, Anhui), and A549 lung cancer cell line was a generous gift from Professor Ting he $\mathrm{Yu}$ at Chongqing Medical University (Chongqing). The cells were grown in Roswell Park Memorial Institute (RPMI) 1640 (for SKOV3) and DMEM (for A549) culture medium containing $10 \% \mathrm{FBS}$ and $1 \%$ penicillin and $1 \%$ streptomycin under a humidified atmosphere in $5 \% \mathrm{CO}_{2}$ at $37^{\circ} \mathrm{C}$. We confirmed that the use of the cell lines has been approved by Ethics Committee of the Second Affiliated Hospital of Chongqing Medical University.

The targeting performance of the TOI_HNPs was tested on SKOV3 (the FR positive) and A549 (the FR negative) by CLSM and flow cytometry (BD Biosciences, San Jose, CA, USA). The cells were seeded into six-well plates $\left(1 \times 10^{6} /\right.$ well $)$ on coverslip. When the cells had grown to $70 \%$, the cells were washed thrice with PBS and replaced with the serumfree medium containing DiI-labeled OI_PNPs or TOI_HNPs ( $8 \mu \mathrm{g} / \mathrm{mL}$ of ICG) at 1 hour incubation. The blockage group was pretreatment with excessive free FA for 1 hour and then added to TOI_HNPs. The free-NPs which had not been taken by cells were removed by washing with PBS thrice. And the cells were fixed with $4 \%$ paraformaldehyde for CLSM detection or harvested for flow cytometry determination.

\section{In vitro therapeutic effect Cytotoxicity of NPs}

The cytotoxicity of NPs on SKOV3 cells was detected by the MTT assay. The cells were seeded into 96-well plates $\left(3 \times 10^{3} /\right.$ well $)$ and incubated for 24 hours, which were co-incubated with different doses of TOI_HNPs for 24 and 48 hours, respectively. Cells without any treatment were used as a control. The cells were washed three times and the old medium was replaced with the serum-free medium containing MTT reagent. Finally, the cell viability was evaluated by the MTT assay (Varioskan Flash; Thermo Fisher Scientific).

The inhibitory effect and mechanism of TOI_HNPs on SKOV3 ovarian cancer cells were further researched. Briefly, The SKOV3 cells were seed into 48 -well plate $\left(1.5 \times 10^{5}\right.$ / well) or 6 -well plate $\left(1 \times 10^{6} /\right.$ well $)$ cultured for 24 hours and replaced the old medium with the serum-free medium containing ICG, TO_HNPs, TI_HNPs, OI_PNPs, and TOI_ HNPs $(8 \mu \mathrm{g} / \mathrm{mL}$ of ICG) at 1 hour incubation, respectively. Then the cells were washed with sterile PBS for three times to remove unbound substrate. After each well was added to new complete medium, the treated cells were exposed to laser (NIR $808 \mathrm{~nm}, 1.5 \mathrm{w} / \mathrm{cm}^{2}, 2$ minutes) and US (1 w/ $\mathrm{cm}^{2}$, 1 minute) successively. Untreated cells in growth media were used as the control. After another 24 hours incubation, the 
relative cell viabilities were measured by the MTT assay. The apoptosis rate in response to different treatment groups was analyzed by flow cytometry.

\section{ROS generation}

Intracellular generation of ROS was measured by fluorescence microscope and fluorescence microplate reader using DCFH-DA as the indicator. The SKOV3 cells were seeded in 48 -well plates $\left(1.5 \times 10^{5} /\right.$ well $)$ or 6 -well plate $\left(1 \times 10^{6} /\right.$ well $)$ cultured for 24 hours and replaced the old medium with the serum-free medium containing different agents (ICG, TO_HNPs, TI_HNPs, OI_PNPs, and TOI_HNPs) at 1 hour incubation. The cells were washed with PBS to remove the excess NPs and exposed to laser plus US. After incubation for another 24 hours, cell culture medium was removed and the serum-free medium including DCFH-DA $(10 \mu \mathrm{M})$ was added and incubated for 1 hour. The green fluorescent signal of $2^{\prime}, 7^{\prime}$-dichlorofluorescein (DCF), which indicated the generation of ROS in the cells, was qualitatively imaged using fluorescence microscopy and quantitatively detected using fluorescence microplate reader (excitation $=485 \mathrm{~nm}$, emission $=528 \mathrm{~nm}$ ).

Simultaneously, the generation of ROS in a cell-free system was measured with a fluorescence microplate reader using SOSG as the indicator; and the substrate solution, containing the mixture of SOSG solution $(5 \mathrm{mM})$ and free ICG, TO_HNPs, OI_PNPs, TI_HNPs, or TOI_HNPs, respectively. Upon laser and US, all groups were incubated for 30 minutes in the dark. The ROS generation was determined by measuring the fluorescence intensity of SOSG with a fluorescence microplate reader $($ excitation $=504 \mathrm{~nm}$, emission $=525 \mathrm{~nm})$.

\section{Western blot analysis}

The proteins were obtained from different treatments of SKOV3 cells using RIPA reagent (Beyotime, Beijing, China) and phenylmethanesulfonyl fluoride (Beyotime, Beijing, China), and the protein concentrations were measured using a BCA protein assay kit (Thermo Fisher Scientific). The protein was separated by $10 \%$ SDS-PAGE and transferred onto a polyvinylidene fluoride (PVDF) membrane (EMD Millipore, Billerica, MA, USA). The membranes were blocked with 5\% nonfat milk for 1 hour and incubated with specific primary antibodies at $4{ }^{\circ} \mathrm{C}$ overnight. The primary antibodies were HIF-1 $\alpha$ (dilution 1:1,000; Affinity Biosciences) and IL-6 (dilution 1:1,000; Affinity Biosciences). The membranes were incubated with secondary antibody glyceraldehyde phosphate dehydrogenase (GAPDH) (dilution 1:500; Affinity Biosciences) for 2 hours at room temperature. The target proteins were visualized using enhanced chemiluminescence solution (Thermo Fisher Scientific).

\section{Statistical analysis}

All experiments were performed triplicate and the data were exhibited as mean \pm SD. The Student's $t$-test was performed for the statistical analysis by GraphPad Prism 6.0 (GraphPad Software, La Jolla, CA, USA). A $P$-value $<0.05$ was considered statistically significant.

\section{Results and discussion Preparation and characterization of NPs} TOI_HNPs, a unique triggered hybrid NP with PLGA core and lipid shell structure (packaging with ICG and PFP-carrying oxygen) was designed for dual-mode imaging and therapeutic effects. These synthesized NPs were more stable than lipid-NPs and had an active targeting ability than PLGA-NPs through folate binding FR. Besides, under laser irradiation, the unique photoluminescence ability of ICG encapsulated into TOI_HNPs endows it with PA imaging performance. And the phase-transition capability of PFP loaded on TOI_HNPs gives it a US imaging ability. Importantly, upon laser irradiation and US exposure, the burst of NPs can release the sensitizers ICG and the PFP-preloaded oxygen for PSDT-induced amplified therapeutic effect by the generated ROS.

The synthesis process of TOI_HNPs is shown in Scheme 1, which was combined with the two-step method and the solvent evaporation technique. The amphiphilic FA-conjugated lipid acted as a shell around the hydrophobic PLGA core which was incorporated ICG and PFP-carrying oxygen. The characterizations of TOI_HNPs are shown in Figure 1.

The TEM analysis confirmed that hybrid NPs had a coreshell structure (Figure 1A), which indicated the successful coating of FA-lipid onto the surface of PLGA polymer.

The physicochemical properties of fabricated NPs are shown in Table 1. All NPs were resuspended in distilled water. The average diameter of the TOI_HNPs, OI_PNPs, and TOI_LNPs measured by DLS was $166.83 \pm 5.54 \mathrm{~nm}$ $(\mathrm{PDI}=0.183 \pm 0.092), 214.50 \pm 10.87 \mathrm{~nm}(\mathrm{PDI}=0.136 \pm 0.051)$, and $340.47 \pm 16.06 \mathrm{~nm}(\mathrm{PDI}=0.149 \pm 0.061)$, respectively. That implies the monodisperse nature of NPs in water solution. The zeta potential absolute values of typically $>30 \mathrm{mV}$ represented the stabilization of particles; $10-30 \mathrm{mV}$ indicating relatively stable and $0-10 \mathrm{mV}$ suggested rapid agglutination of particle instability. ${ }^{27}$ The zeta potential values of TOI_HNPs, OI_PNPs, and TOI_LNPs were $-(30.57 \pm 1.36),-(24.04 \pm 1.17)$, and $-(17.50 \pm 1.65) \mathrm{mV}$, respectively. Obviously, the result showed that TOI_HNPs 


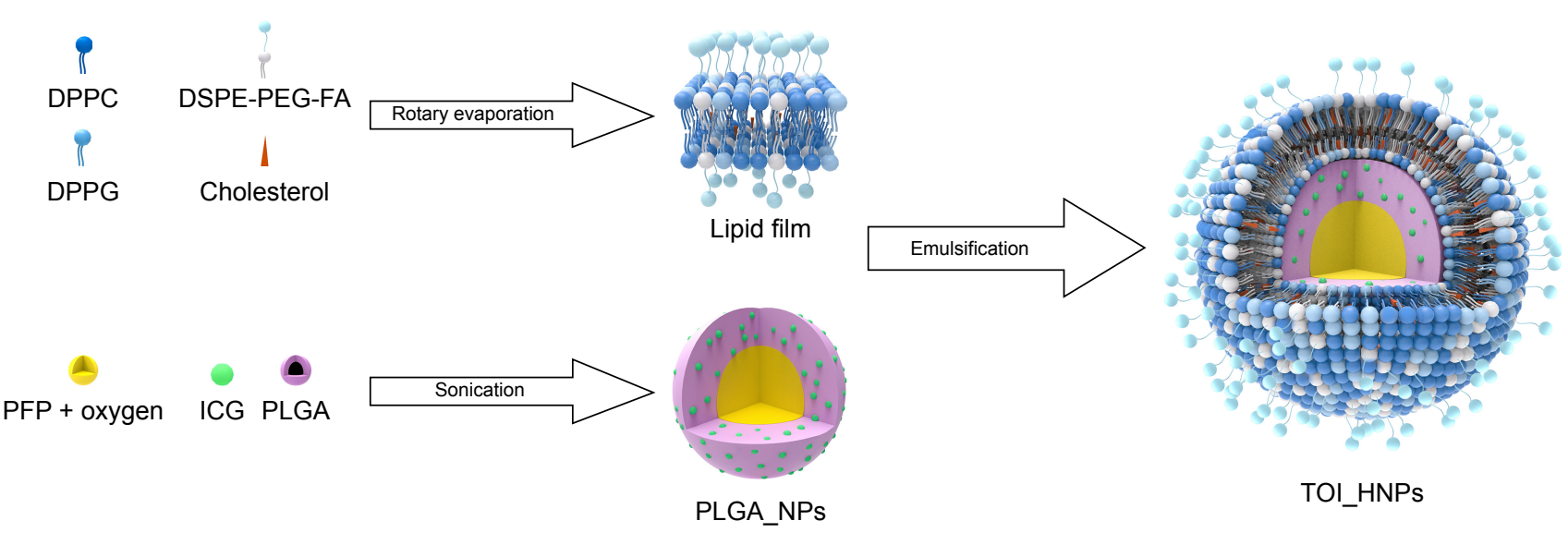

Scheme I Schematic illustration of the synthesis and structure of TOI_HNPs.

Abbreviations: DPPC, phosphatidylcholine;DPPG, I,2-dipalmitoyl-sn-glycero-3-phospho-(I'-rac-glycerol); DSPE-PEG-FA, I,2-distearoyl-sn-glycero-3-phosphoethanolamine$\mathrm{N}$-[folate(polyethyleneglycol)-2000]; ICG, indocyanine green; LPHNPs, lipid-polymer hybrid nanoparticles; PFP, perfluoropentane; PLGA_NPs, poly (lactic-co-glycolic acid) nanoparticles; TOI_HNPs, folate-targeted LPHNPs-loaded ICG/PFP-carrying oxygen.

may be more stable than the other two particles. Besides, the size and zeta potential had no significant difference between the TO_HNPs and TI_HNPs. The drug EE and drug LE played a critical role in the clinical application. The EE of ICG incorporated in TOI_HNPs was $75.29 \% \pm 2.92 \%$, while the LE was $1.02 \% \pm 0.04 \%$, which was demonstrated that the method can successfully package ICG into the nanocarriers with a higher encapsulation rate than OI_PNPs which was $62.48 \% \pm 3.39 \%$. It was reported that the solvent evaporation method that loaded hydrophilic molecules usually suffered from low encapsulation efficiencies and the encapsulated hydrophilic ICG could rapidly diffuse into the external aqueous during formation. ${ }^{12,24,28-30}$ Evidently, the encapsulation efficiency of ICG have been improved in the LPHNP through combination the two-step method and the solvent evaporation technique

The phase-transformation of TOI_HNPs was observed under the optical microscope by laser irradiation or heated.
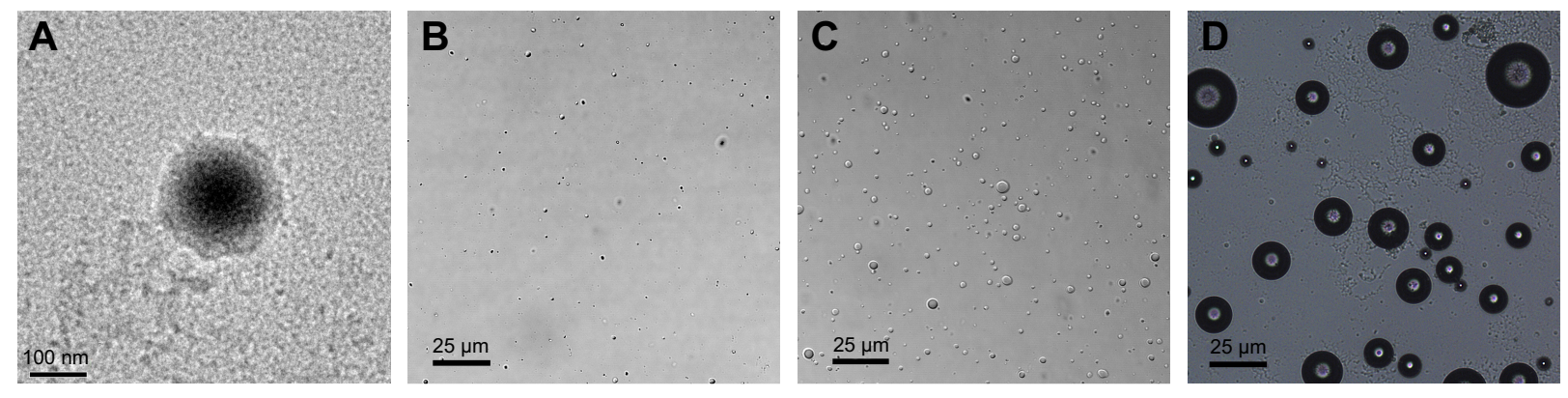

E

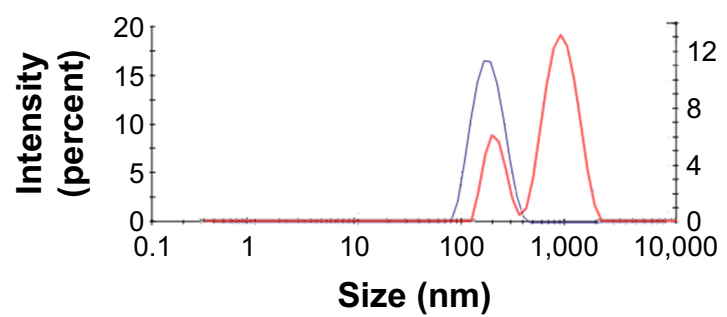

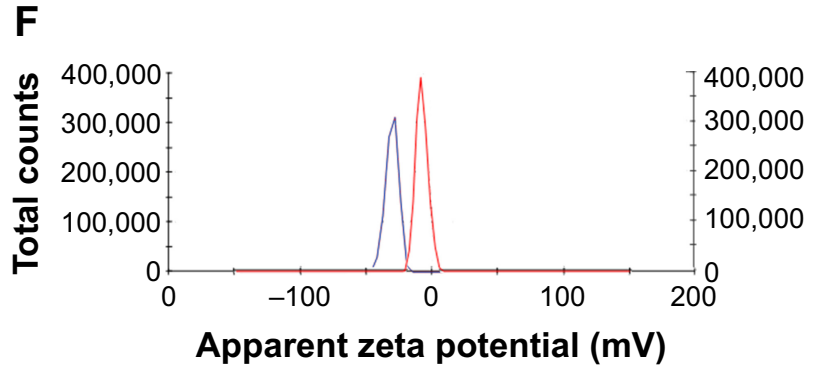

Figure I Characterization of TOI_HNPs.

Notes: (A) TEM image of TOI_HNPs. (B, C) Optical microscopy images of TOI_HNPs and phase-transited TOI_HNPs after laser irradiation. (D) Optical microscopy image of TOI_HNPs heated at $49^{\circ} \mathrm{C}$ for 10 seconds. (E, F) Size distribution and zeta potential of TOI_HNPs before (the blue line) and after (the red line) laser irradiation measured by DLS.

Abbreviations: DLS, dynamic light scattering; ICG, indocyanine green; PFP, perfluoropentane; LPHNPs, lipid-polymer hybrid nanoparticles; TEM, transmission electron microscope; TOI_HNPs, folate-targeted LPHNPs-loaded ICG/PFP-carrying oxygen. 
Table I Physicochemical property of the fabricated NPs

\begin{tabular}{l|l|l|l|l|l}
\hline Groups & Size $(\mathbf{n m})$ & PDI & Zeta potential $(\mathbf{m V})$ & EE $(\%)$ & LE $(\%)$ \\
\hline TOI_HNPs & $166.83 \pm 5.54$ & $0.183 \pm 0.092$ & $-30.57 \pm 1.36$ & $75.29 \pm 2.92$ & $1.02 \pm 0.04$ \\
OI_PNPs & $214.50 \pm 10.87$ & $0.136 \pm 0.05 \mathrm{I}$ & $-24.04 \pm 1.17$ & $62.48 \pm 3.39$ & $1.84 \pm 0.10$ \\
TOI_LNPs & $340.47 \pm 16.06$ & $0.149 \pm 0.06 \mathrm{I}$ & $-17.50 \pm 1.65$ & $82.50 \pm 2.84$ & $2.20 \pm 0.08$ \\
TO_HNPs & $170.07 \pm 1.63$ & $0.083 \pm 0.063$ & $-28.33 \pm 1.50$ & - & - \\
TI_HNPs & $171.53 \pm 4.68$ & $0.056 \pm 0.008$ & $-27.27 \pm 1.64$ & - & - \\
\hline
\end{tabular}

Note: Values are represented as mean \pm SD $(n=3)$.

Abbreviations: EE, encapsulation efficiency; ICG, indocyanine green; LE, loading efficiency; NPs, nanoparticles; PDI, polydispersity; PFP, perfluoropentane; LPHNPs, lipidpolymer hybrid nanoparticles; TOI_HNPs, folate-targeted LPHNPs-loaded ICG/PFP-carrying oxygen; OI_PNPs, PLGA NPs-encapsulated ICG and PFP-carrying oxygen; PLGA, poly (lactic-co-glycolic acid); TOI_LNPs, folic-targeted lipid NPs-encapsulated ICG and PFP-carrying oxygen; TO_HNPs, folate-targeted LPHNPs-loaded PFP-carrying oxygen; TI_HNPs, folate-targeted LPHNPs-loaded ICG.

The optical microscopy images (Figure 1B) of TOI_HNPs before irradiation showed that NP was a uniform spherical shape and nanoscale. It was found that particles enlarged by ODV principles after $808 \mathrm{~nm}$ laser irradiation (Figure 1C). Sequentially, with US exposure, the enlarged microbubbles ruptured (data are shown). In addition, such "small-to-big" phenomenon can also be observed if the temperature rose to $49^{\circ} \mathrm{C}$ (Figure 1D) and a large number of microbubbles were generated. After NIR irradiation, the size distribution significantly increased and the zeta potential absolute value slightly decreased (Figure 1E and F). Thus, the liquid-to-gas transition (vaporization) study demonstrates that the obtained hybrid NPs-encapsulated PFP could act as a phase-change agent triggered by laser. The boiling point of PFP is $29^{\circ} \mathrm{C}$, and the phase transition of fabricating TOI_HNPs occurred when the temperature raised to $49^{\circ} \mathrm{C}$. The result showed that PFP encased in TOI_HNPs had a significantly higher boiling point than the free liquid state. This inconsistency was probably owing to the assembly of these materials. ${ }^{19,31}$ The capability of US imaging was predominantly attributed to the cavitation effect of stable bubbles from the NPs. ${ }^{32}$
This in vitro phase-transition ability of TOI_HNPs implied the US imaging potential.

Furthermore, the essential properties of NP were also detected as shown in Figure 2. The absorption spectra (Figure 2A) and fluorescence spectrum peaks (Figure 2B) of TOI_HNPs, OI_PNPs, and TOI_LNPs were located at $780 \mathrm{~nm}$ and $808 \mathrm{~nm}$ as the free ICG solution. It showed that the preparation of NPs did not change the optical properties of ICG. Figure $2 \mathrm{C}$ and D exhibited the changes in the normalized absorbance and fluorescence intensity of NPs and free ICG solution measured within 15 days at 3 days interval. The TOI_HNPs absorbance was decreased to $75.4 \%$ of its initial intensity, while the OI_PNPs, TOI_LNPs, and free ICG decreased to $56.2 \%, 35.4 \%$, and $12.5 \%$, respectively. Besides, the fluorescence intensity of TOI_HNPs remained at $62.7 \%$, while the fluorescence of OI_PNPs, TOI_LNPs, and free ICG were reduced to $35.6 \%, 14.8 \%$, and $4.0 \%$, respectively. Regardless TOI_HNPs, OI_PNPs, and TOI_LNPs loaded ICG, the absorbance and fluorescence intensity of the NPs were more stable than free ICG, among which the stability of TOI_HNPs is the best.
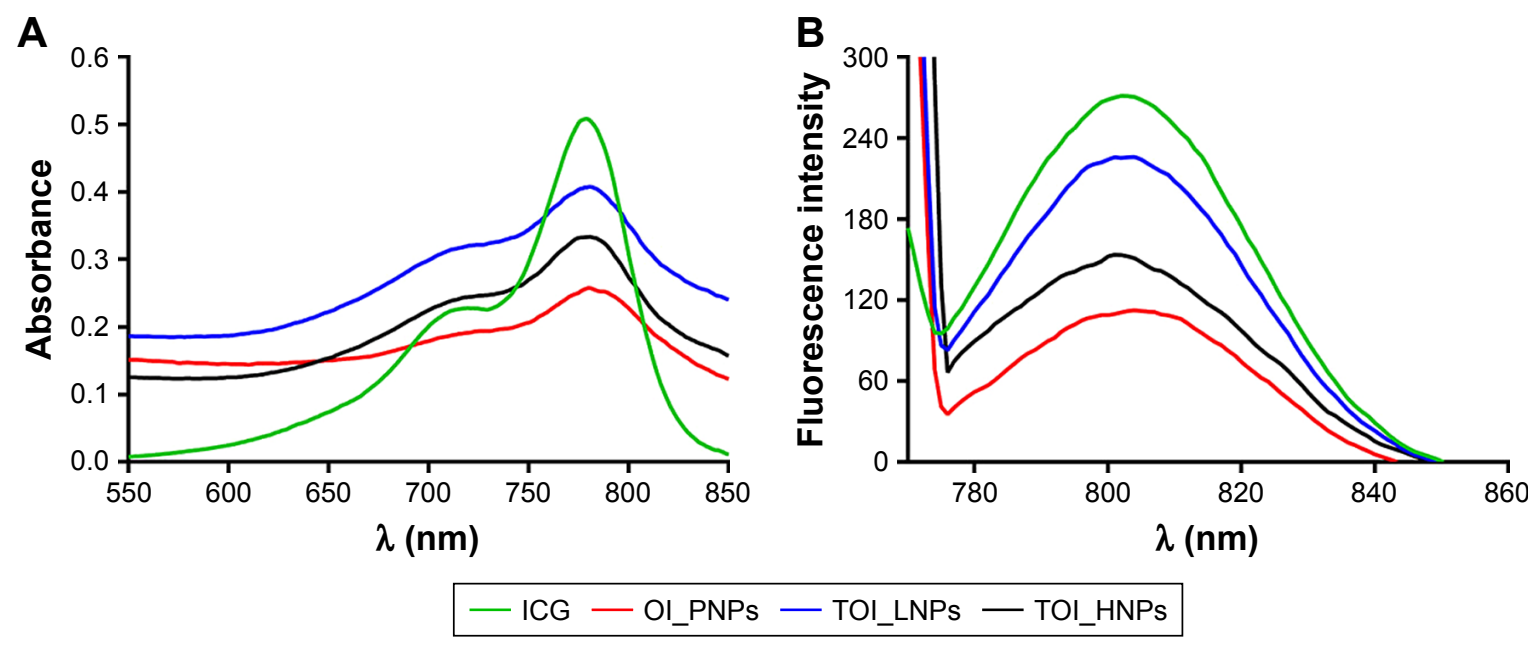

Figure 2 (Continued) 
C

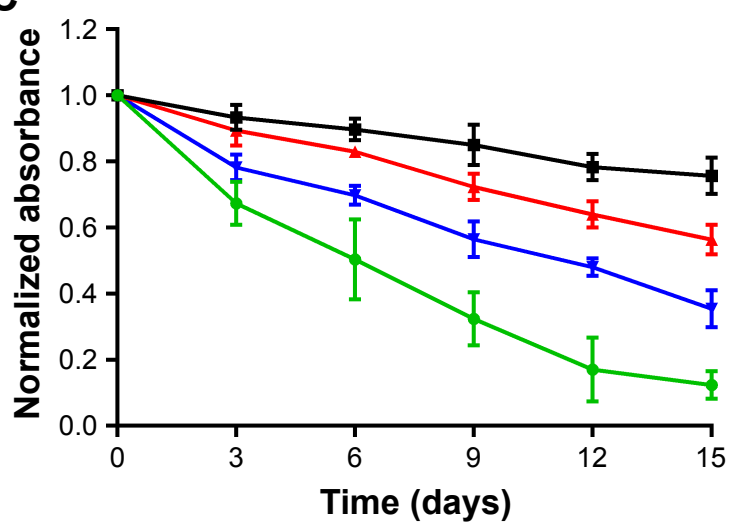

D

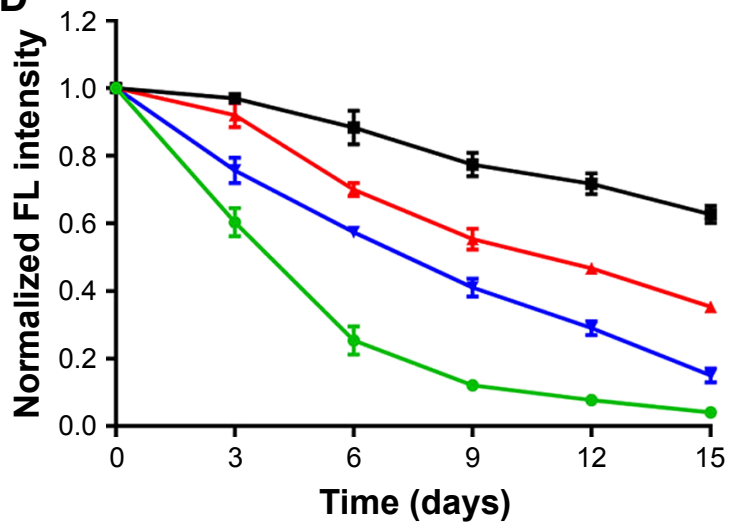

$\rightarrow$ ICG $\leftarrow$ OI_PNPs $\rightarrow$ TOI_LNPs $\rightarrow$ TOI_HNPs

E

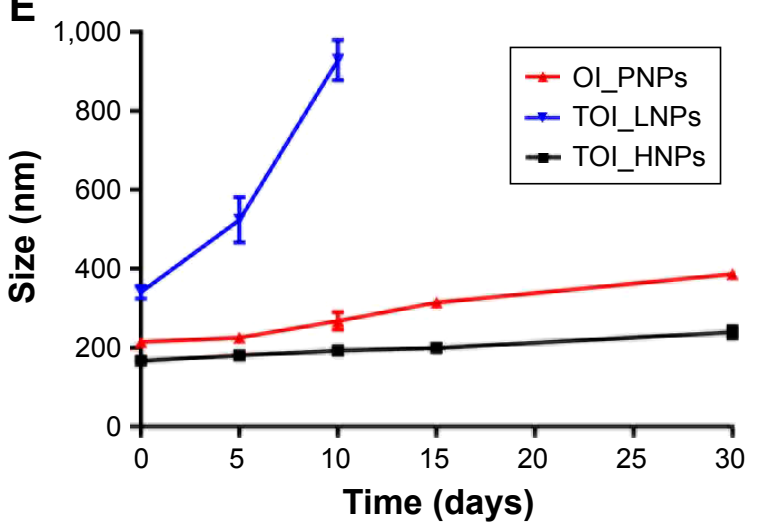

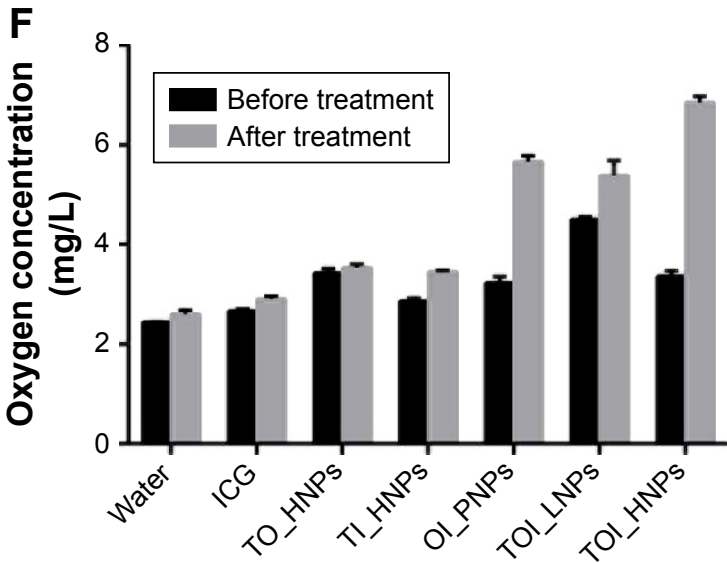

G

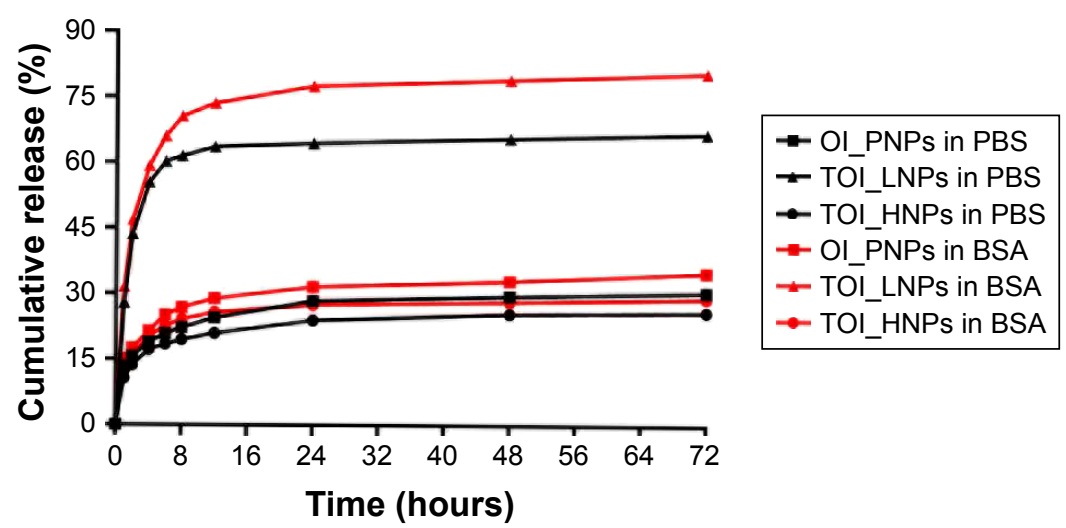

Figure 2 Comparison of essential properties for developed NPs.

Notes: (A, B) Absorption and fluorescence spectra of free ICG, OI_PNPs, TOI_LNPs, and TOI_HNPs. (C, D) Normalized absorption and fluorescence intensity of free ICG, OI_PNPs, TOI_LNPs, and TOI_HNPs every 3 days. (E) The size stability of OI_PNPs, TOI_LNPs, and TOI_HNPs at different time intervals. (F) The change of the dissolved oxygen concentration levels for prepared solutions before and after laser plus US treatment. (G) Release profile of ICG from OI_PNPs, TOI_LNPs, and TOI_HNPs in PBS and in $\mathrm{BSA}$ at $37^{\circ} \mathrm{C}$ and $100 \mathrm{rpm}$.

Abbreviations: ICG, indocyanine green; LPHNPs, lipid-polymer hybrid nanoparticles; NPs, nanoparticles; OI_PNPs, PLGA NPs-encapsulated ICG and PFP-carrying oxygen; PFP, perfluoropentane; PLGA, poly (lactic-co-glycolic acid); TOI_HNPs, folate-targeted LPHNPs-loaded ICG/PFP-carrying oxygen; TOI_LNPs, folic-targeted lipid NPs-encapsulated ICG and PFP-carrying oxygen; US, ultrasound; FL, fluorescence.

In addition, NPs stability in solution was an important criterion for their utility as drug carriers. We investigated the stability of the particle size changes after 1 month storage at $4^{\circ} \mathrm{C}$. As shown in Figure 2E, the size of TOI_HNPs was slightly increased from 166.8 to $239.4 \mathrm{~nm}$. And the size of OI_PNPs was increased from 214.5 to $386.3 \mathrm{~nm}$ within 30 days. It is worth noting that the size of TOI_LNPs increased from 340.5 to $524.0 \mathrm{~nm}$ within 5 days and the monodisperse 
state almost disappeared on the tenth day. Compared with OI_PNPs and TOI_LNPs, core-shell NPs were more stable. A possible mechanism was that lipid shell played a protective role in PLGA NPs, while PLGA-core played a stabilizing role in lipid nanocarriers. ${ }^{22-24}$ Therefore, TOI_HNPs can be stored for a long time without a spontaneous phase transition.

The oxygen release capacity of TOI_HNPs was tested by measuring the oxygen concentration changes after treatment (laser plus US). As shown in Figure 2F, the concentration of the dissolved oxygen in TOI_HNPs, TOI_LNPs, and OI_ PNPs increased from 3.36 to $6.85 \mathrm{mg} / \mathrm{L}, 4.50$ to $5.38 \mathrm{mg} / \mathrm{L}$, and 3.23 to $5.66 \mathrm{mg} / \mathrm{L}$, respectively. These implied that PFP was successfully encapsulated in NPs. Besides, the basal oxygen level in TOI_HNPs, TOI_LNPs, OI_PNPs, and TO_HNPs groups were higher than that in degassed water. This may be due to the PFP-carrying oxygen and released $\mathrm{O}_{2}$ by simple diffusion through concentration gradient, ensuring the timely oxygen supply to hypoxia surroundings. ${ }^{28}$ In particular, the TOI_LNPs were maintained at a high concentration compared with the other groups, for its instability causes spontaneous rupture. Moreover, compared with the TO_HNPs and TI_HNPs groups, the change of oxygen concentration in TOI_HNPs group is more obvious after treatment, which may be due to the presence of ICG in nanoagents for PSDT mediating the phase transition and rupture of NPs to release oxygen from PFP. Therefore, this data demonstrated that the encapsulated PFP in the NPs could be the excellent oxygen reservoir and useful for delivering oxygen.

The release profiles of ICG from TOI_HNPs, OI_PNPs, and TOI_LNPs in PBS ( $\mathrm{pH}=7.4$ ) and 10\% BSA solution at $37^{\circ} \mathrm{C}$ and $100 \mathrm{rpm}$ are shown in Figure 2G. Drug release occurred in an initial "burst" release phase and subsequent slow release. ${ }^{7}$ In detail, the cumulative ICG release of TOI_LNPs in PBS and BSA reached to $66.68 \%$ and $80.59 \%$, respectively. Correspondingly, the release of OI_PNPs was $30.51 \%$ in PBS and $34.97 \%$ in BSA. However, TOI_HNPs showed 25.95\% (in PBS) and 29.05\% (in BSA) accumulative release after 72 hours. All the results indicated that lipid-polymer hybrid NPs can be used as remarkable carries for drug delivery, both in vitro and in vivo. This might associate with the lipid layer at the surface of the PLGA core which acted as a molecular fence that helped to retain the ICG inside the NPs.

\section{Dual-mode imaging in vitro}

The US and PA imaging properties of TOI_HNPs were tested in an agar-gel model. The results of US imaging (B mode), contrast-enhanced ultrasound (CEUS), and semiquantitative EI about degassed water, free ICG, TO_HNPs, and TOI_HNPs $(8 \mu \mathrm{g} / \mathrm{mL}$ of ICG) solution with and without laser irradiation are shown in Figure 3A. There were no obvious changes in B mode or CEUS images in the water group and free ICG group. In the TO_HNPs, after laser irradiation, there were slight changes to the B mode and CEUS images. Interestingly, in the TOI_HNPs group, the contrast-enhanced imaging can be observed and the EI in B mode and CEUS increased from $8.19 \pm 0.91$ to $81.88 \pm 10.73$ and from $4.87 \pm 0.51$ to $49.13 \pm 0.91$, respectively (Figure $3 \mathrm{~B}$ and $\mathrm{C}$ ). These changes were attributed to the liquid-gas phase transition of PFP stimulated by laser-induced photosensitizers. The ODV principle enabled the nanosized agent to generate large amounts of micrometer-sized bubbles through laser stimulated optical absorbs, which can significantly enhance the echo signals of the ultrasonography since they can function as the reflectors for an US by the resonance at the diagnostic frequency. Meanwhile, the rapid volume expansion during the ODV also generated PA signal with large amplitude. ${ }^{17,33}$

The US mode and PA mode (US images and PA images) of TOI_HNPs suspension are shown in Figure 3D. There was significant PA contrast imaging in TOI_HNPs after NIR irradiation, while TO_HNPs showed no obvious PA contrast enhancement, and even the ICG PA signal was weakened. This was consistent with semi-quantitative results (Figure 3E and F). A large amount of ICG release under NIR irradiation causes the PA average value of TOI_HNPs group to increase from $0.145 \pm 0.016$ to $1.526 \pm 0.090$. Interestingly, the PA signal intensity decreased from $0.274 \pm 0.015$ to $0.060 \pm 0.003$ in ICG group. This change was ascribed to photobleaching of the free ICG after irradiation. Thus, it has demonstrated that TOI_HNPs could considerably enhance US and PA imaging as an ideal contrast agent.

\section{Targeting effect}

Efficient cellular uptake and excellent intracellular distribution of TOI_HNPs were prerequisite for improving PSDT to kill cancer cells. The targeting efficiency of TOI_HNPs and OI_PNPs was systematically investigated by CLSM. TOI_HNPs and OI_PNPs could effectively target to SKOV3 ovarian cancer cells after incubation for 1 and 4 hours, respectively (data not shown). As shown in Figure 4A, there was strong red fluorescence (DiI-loaded NPs) in the TOI_HNPs group.Comparatively, OI_PNPs only expressed weak red fluorescence after 1 hour co-incubation. Moreover, TOI_HNPs showed the insufficient intracellular uptake after the target site on the SKOV3 cell membrane was competitively bound by free FA. Consistent with flow cytometry analysis, the difference of fluorescence value between TOI_HNPs and other groups was statistically significant 
A

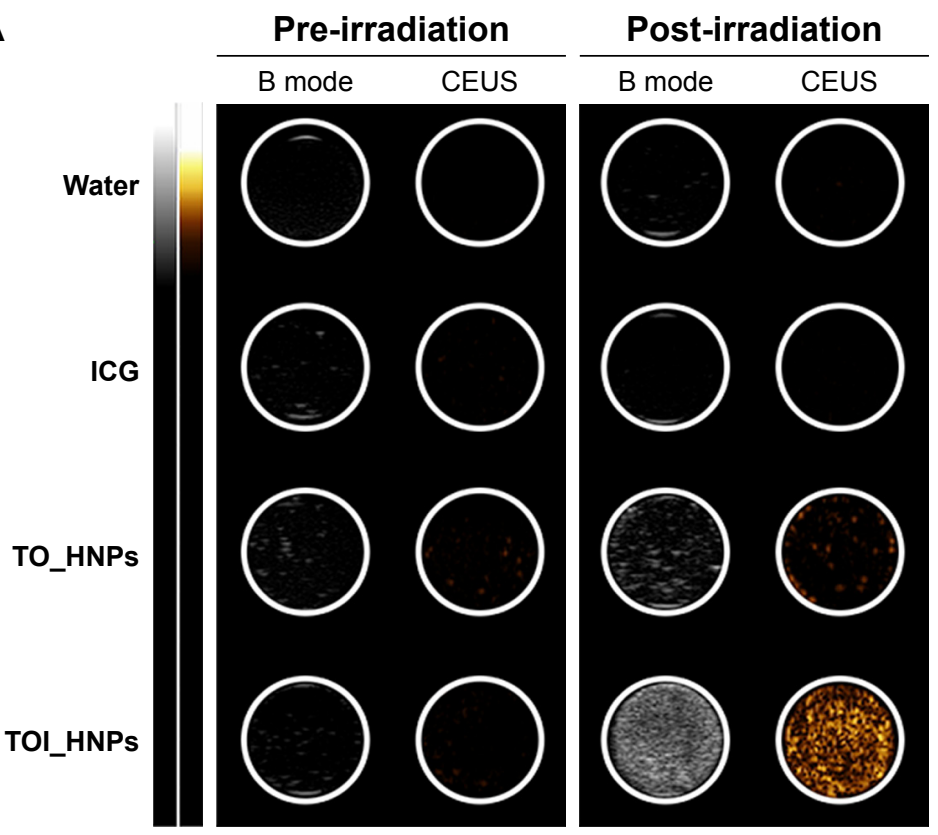

D

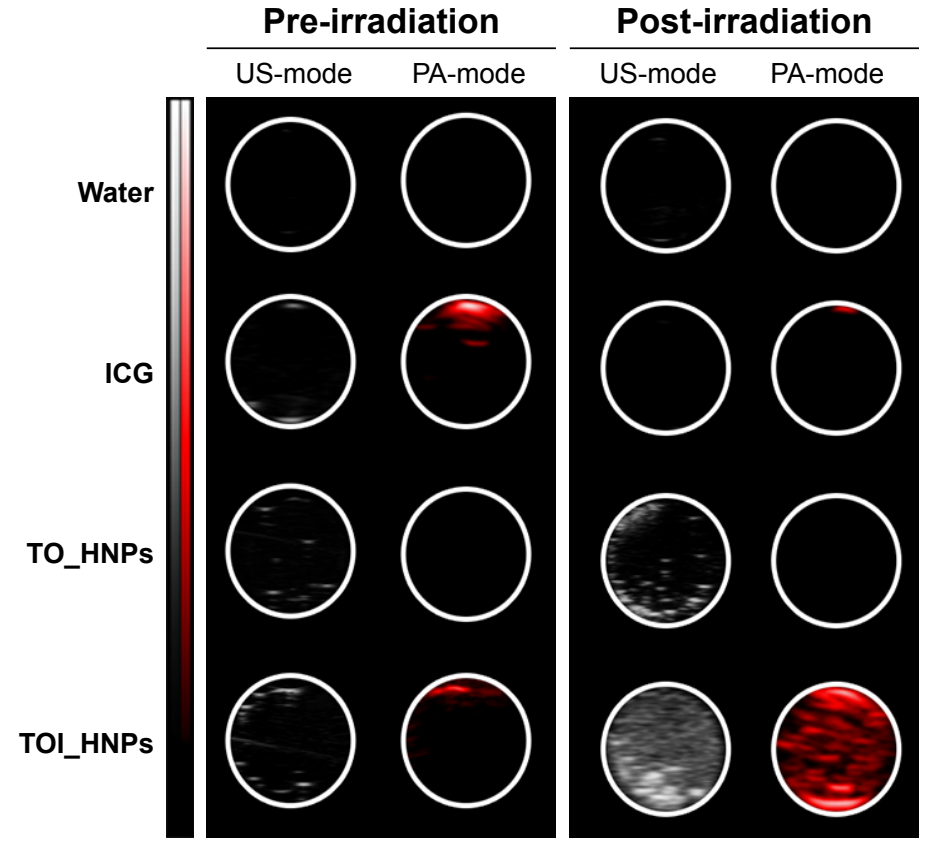

B

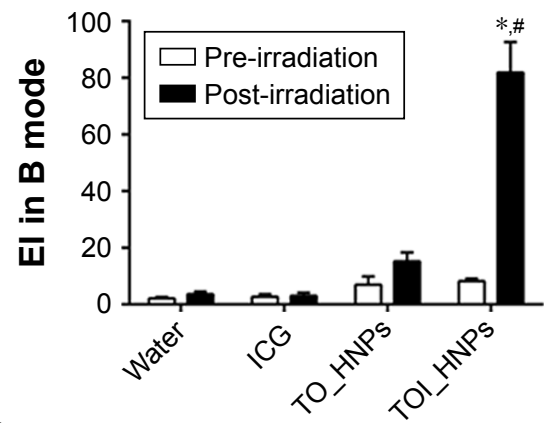

C

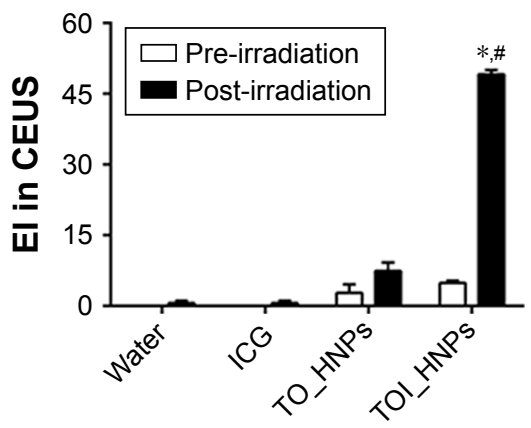

E

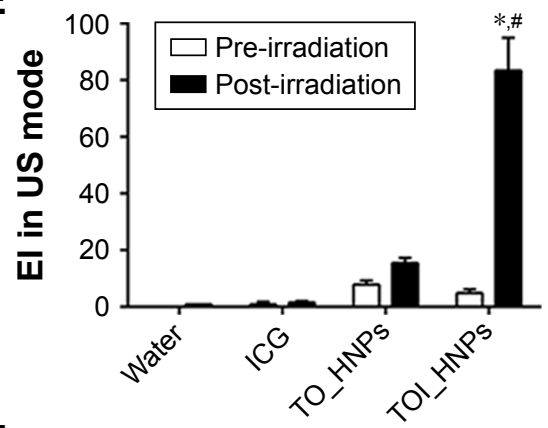

$\mathbf{F}$

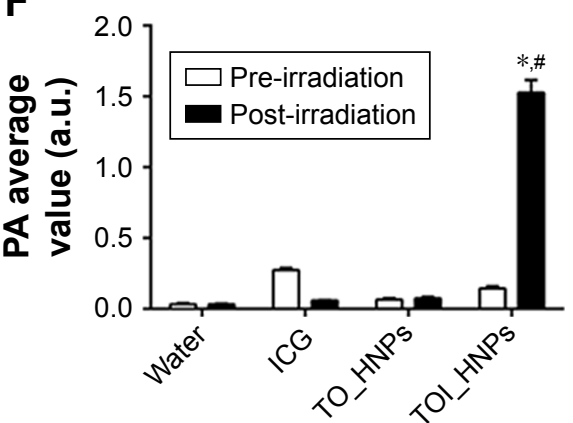

Figure 3 Dual-mode imaging in vitro agar-gel model.

Notes: (A) B mode and CEUS imaging of particles before and after laser irradiation for degassed water, free ICG, TO_HNPs, and TOI_HNPs. (B, C) The semi-quantitative of El in B mode and CEUS before and after laser irradiation. (D) US mode and PA mode imaging for the same groups before and after laser irradiation. (E, F) The semiquantitative of El in US mode and PA average value in PA mode before and after laser irradiation. TOI_HNPs of post-irradiation compared with the pre-irradiation, $* P<0.05$; after laser irradiation, TOI_HNPs compared with the other groups at the same conditions, ${ }^{~} P<0.05$.

Abbreviations: CEUS, contrast-enhanced ultrasound; El, echo intensity; ICG, indocyanine green; LPHNPs, lipid-polymer hybrid nanoparticles; PFP, perfluoropentane; TOI_HNPs, folate-targeted LPHNPs-loaded ICG/PFP-carrying oxygen; US, ultrasound; TO_HNPs, folate-targeted LPHNPs-loaded PFP-carrying oxygen.

(Figure 4B and C). In addition, compared with the SKOV3 cells (FR positive), the A549 cell (FR negative) group showed inadequate intracellular uptake of both TOI_HNPs and OI_PNPs group. Further, there was no significant difference (Figure 4D and E). Therefore, there was such an uptake difference between TOI_HNPs and OI_PNPs on ovarian cancer cell due to FA targeting FR rather than the endocytosis pathway. 


\section{Therapeutic effect}

The cytotoxic effects on SKOV3 cells were investigated. Primarily, the safety of TOI_HNPs in vitro was determined by MTT assay. The results showed that there was no significant cytotoxicity at 24 and 48 hours incubation of SKOV3 cells with NPs in $0-8 \mu \mathrm{g} / \mathrm{mL}$ of ICG concentration, as shown in Figure 5A. The difference between each group had no statistical significance. However, with increasing concentration and longer incubation time, a number of cells died. The decrease in cell viability after high doses of NPs incubation might involve a combination of complicated processes including cellular stress generation, induction of autophagy,

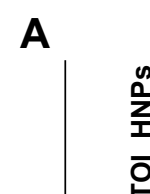
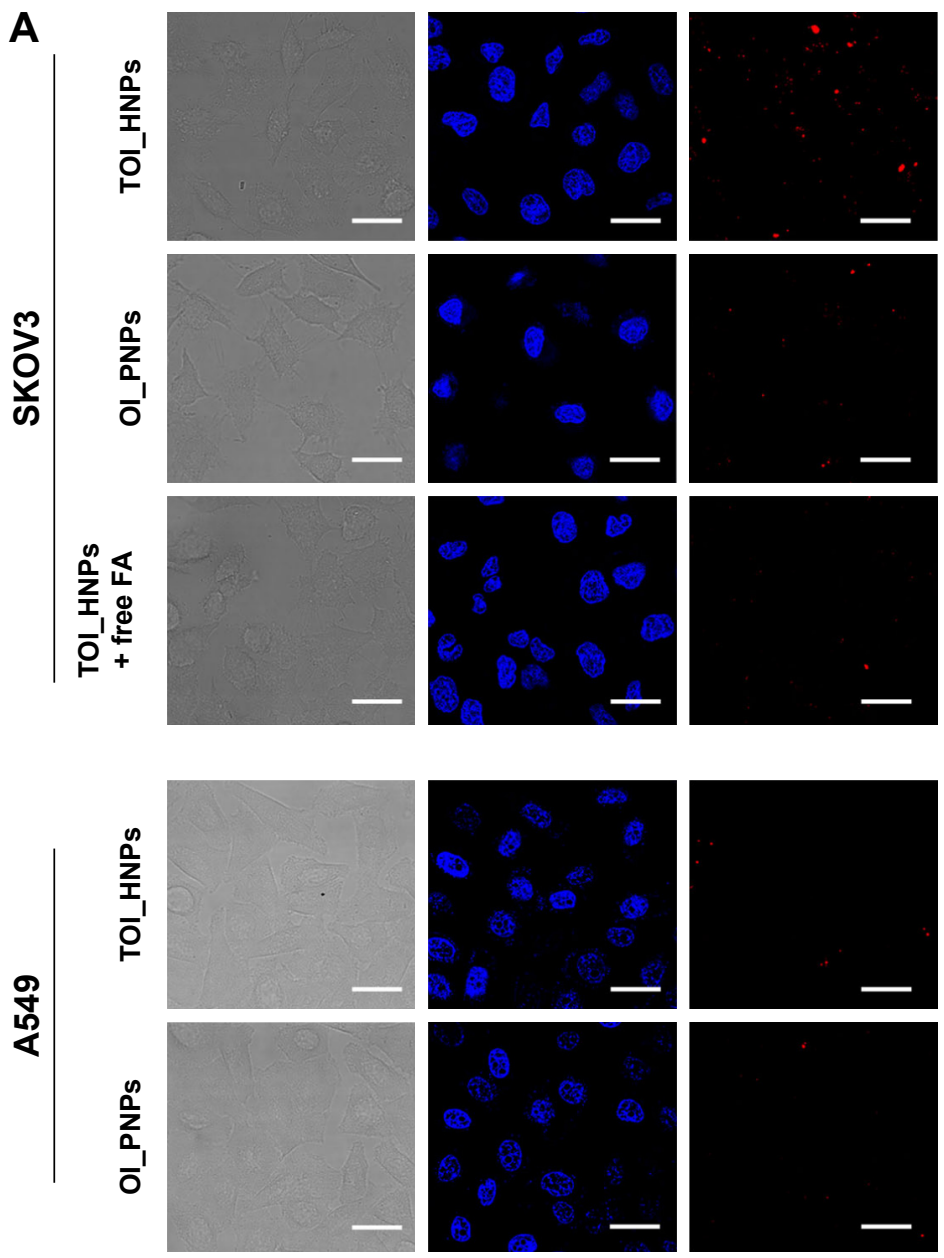
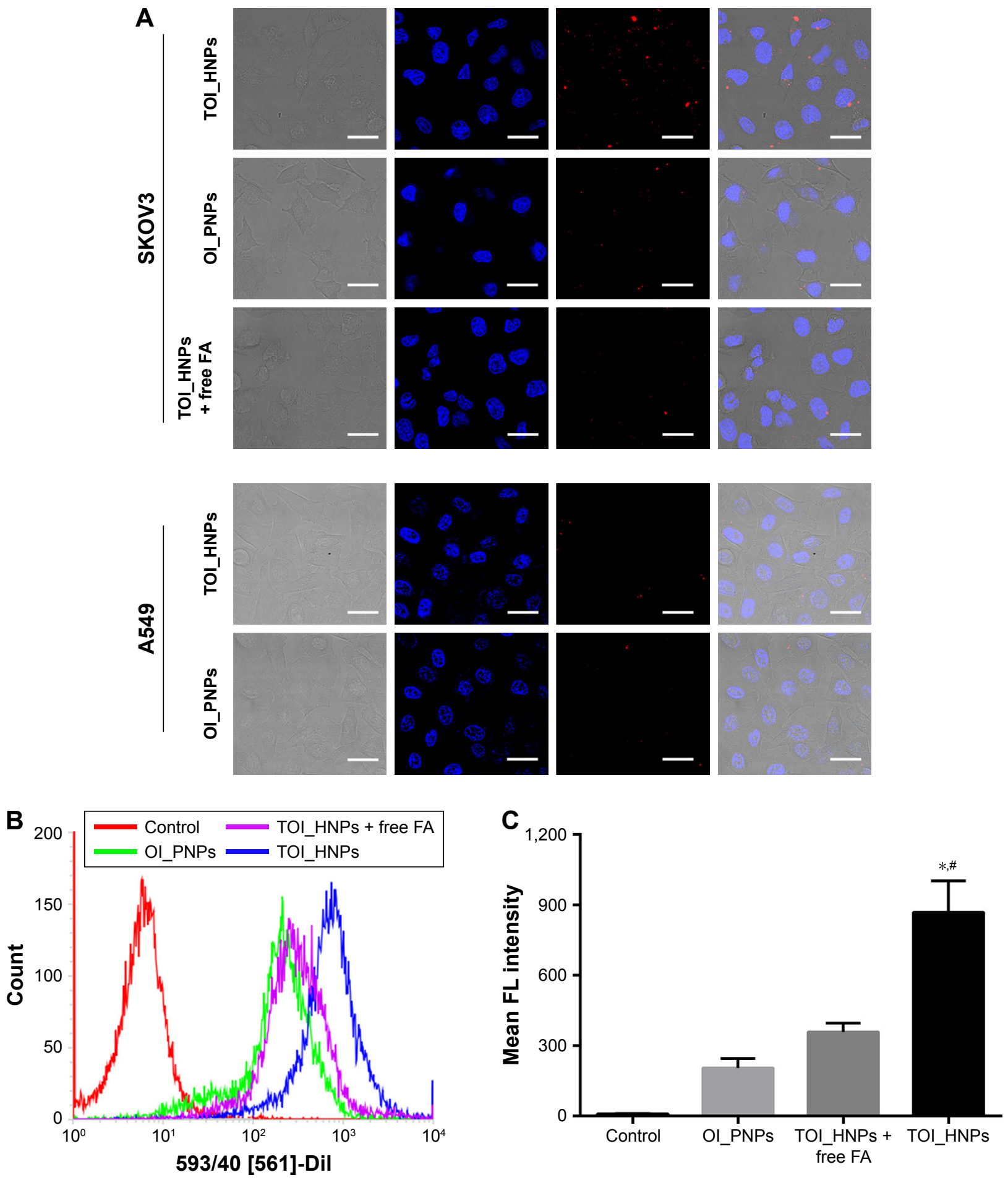

Figure 4 (Continued) 

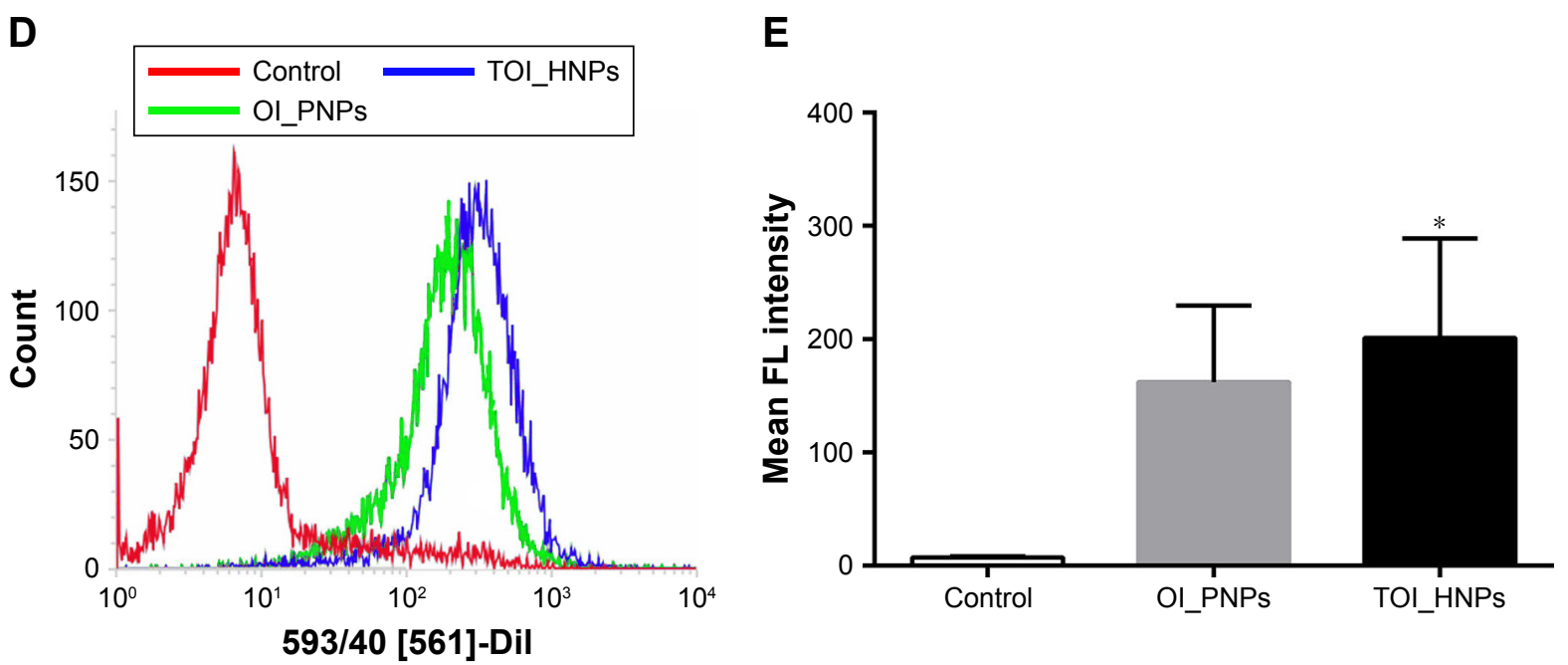

Figure 4 Cellular uptake of nanoparticles by SKOV3 cells (FR positive) and A549 cells (FR negative).

Notes: (A) Fluorescence confocal microscopy images of SKOV3 cells incubated with TOI_HNPs, OI_PNPs, and TOI_HNPs plus free FA for I hour. A549 cells incubated with TOI_HNPs and OI_PNPs for I hour. Scale bar is $25 \mu \mathrm{m}$. (B, C) Flow cytometric analysis of mean fluorescence intensity for SKOV3 cells. (D, E) Flow cytometric analysis of mean fluorescence intensity for A549 cells. Compared with control, $* P<0.05$; compared with other groups, ${ }^{*} P<0.05$.

Abbreviations: FA, folic acid; ICG, indocyanine green; LPHNPs, lipid-polymer hybrid nanoparticles; PFP, perfluoropentane; TOI_HNPs, folate-targeted LPHNPs-loaded ICG/PFP-carrying oxygen; OI_PNPs, PLGA NPs-encapsulated ICG and PFP-carrying oxygen; PLGA, poly (lactic-co-glycolic acid); FL, fluorescence.

interferation of cytoskeleton architecture, and changes in membrane integrity. ${ }^{34-36}$ And also, at high intracellular NP concentrations, direct interaction of NPs with cells is essential. ${ }^{37}$ There was a certain intracellular "safe" threshold for TOI_HNPs. Therefore, for TOI_HNPs an ICG concentration of $8 \mu \mathrm{g} / \mathrm{mL}$ was used in our studies.

Figure 5B shows that the cell viability of TOI_HNPs + PSDT group was $(16.39 \% \pm 2.58 \%)$, the OI_PNPs + PSDT group and TI_HNPs + PSDT group was $(41.58 \% \pm 2.10 \%)$ and $(53.85 \% \pm 4.58 \%)$, respectively. However, no obvious cytotoxicity was observed in ICG + PSDT or TO_HNPs + PSDT groups $(97.70 \% \pm 2.35 \%)$ and $(98.49 \% \pm 2.03 \%)$, respectively. It showed that TOI_HNPs-mediated PSDT significantly inhibited cell viability when compared with other groups. Moreover, the equal concentration of ICG group and TO_ HNPs group had less effect on cell viability after exposured laser plus US. The apoptosis rate by flow cytometry analysis was consistent with the results of MTT assay. Figure $5 \mathrm{C}$ and D exhibits that the apoptosis rate of TOI_HNPs + PSDT group was $(81.58 \% \pm 7.68 \%)$, while the apoptosis rate of OI_PNPs + PSDT group and TI_HNPs + PSDT group was only $(52.04 \% \pm 14.66 \%)$ and $(43.26 \% \pm 11.10 \%)$, respectively. Compared with OI_PNPs, TOI_HNPs contained the same concentration of ICG that leads to more cell apoptosis due to the active target. Furthermore, although TI_HNPs without oxygen encapsulation could also target cells by FA bind to FR, they could not reach the same level of apoptosis as TOI_HNPs, which suggested that TOI_HNPs induced-PSDT required ICG and oxygen. PSDT is a new and promising anti-cancer therapy, which stimulates sensitizers by light plus US to enhance anticancer effects. ${ }^{38}$ Indeed, previous studies have explored that, compared with the monotherapy (PDT or SDT), PSDT decreased the sensitizer dosage and light/US energy that can further reduce the side effects. ${ }^{7,39-43}$ Moreover, our results demonstrated that both laser and US were essential elements for evaporation and destruction of TOI_HNPs. Thus, we did not test the therapeutic efficiency of TOI_HNPs + PDT and TOI_HNPs + SDT.

The underlying mechanism of PSDT effect was unclear, but many investigations have demonstrated that increased ROS production may play an important role in this process. ${ }^{38,39,44,45}$ The intracellular ROS generation capability of TOI_HNPs was further qualitatively observed by fluorescence microscopy and quantified by fluorescence microplate reader. As shown in Figure 6A, DCFH-DA acted as an intracellular ROS probe, which could be rapidly oxidized to DCF with green fluorescence. Under $808 \mathrm{~nm}$ laser irradiation and US exposure, SKOV3 cells treated with TOI_HNPs showed obviously green fluorescence. In contrast, only slightly green fluorescence was observed in the cells treated with OI_PNPs and TI_HNPs, and weak fluorescence was observed in ICG and TO_HNPs. As confirmed by quantitative experiments, the TOI_HNPs, TI_HNPs, and OI_PNPs significantly increased intracellular DCF fluorescence after treatment. In TOI_HNPs + PSDT group, the DCF fluorescence increased at $(244.27 \% \pm 29.11 \%)$, higher than other groups (Figure 6B). It confirmed that TOI_HNPs-mediated PSDT can greatly generate ROS to kill cells. 
A

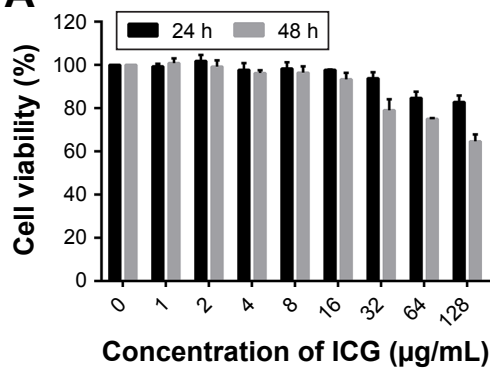

Concentration of ICG $(\mu \mathrm{g} / \mathrm{mL})$

D
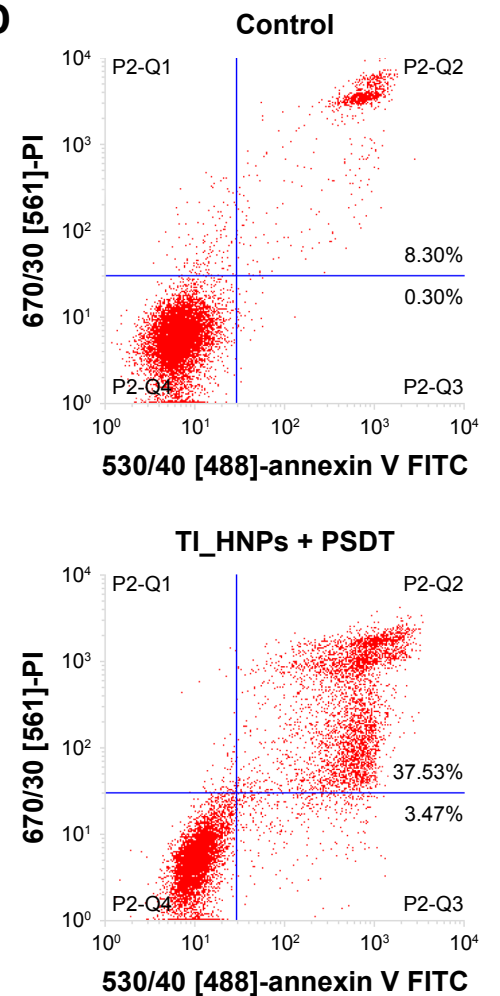

B

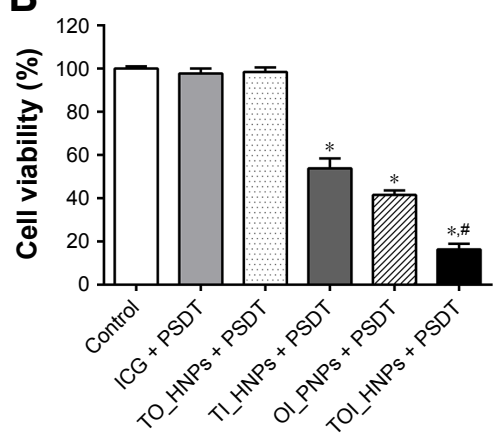

ICG + PSDT
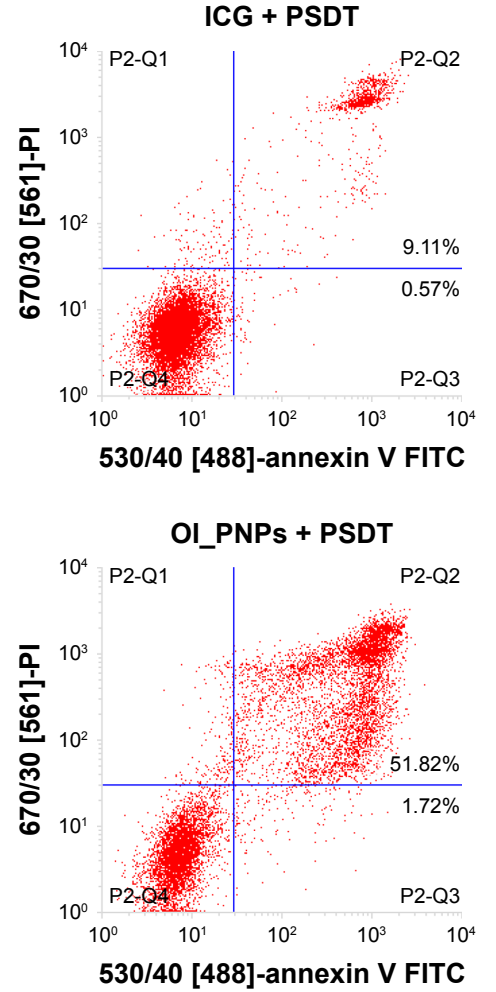
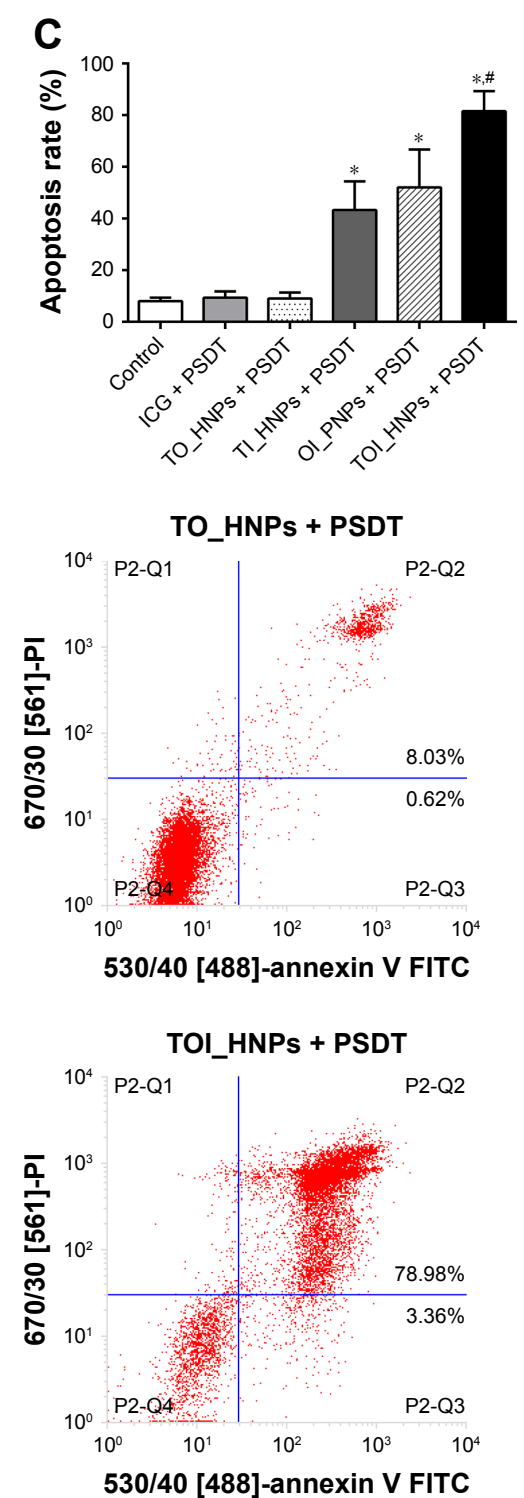

Figure $\mathbf{5}$ Therapeutic effect in SKOV3 cells after different treatments.

Notes: (A) Cytotoxicity of TOI_HNPs using the MTT assay at a concentration of ICG for $0-128 \mu \mathrm{g} / \mathrm{mL}$. (B) Cell viability of SKOV3 cells under different treatments was determined by the MTT assay $(\overline{8} \mu \mathrm{g} / \mathrm{mL}$ of ICG). (C, D) Apoptosis detected by flow cytometry 24 hours after different treatments. Compared with control, $* P<0.05$; compared with other groups, ${ }^{\#} P<0.05$.

Abbreviations: ICG, indocyanine green; MTT, 3-(4,5-dimethylthiazol-2-yl)-2,5-diphenyltetrazoliumbromide; PFP, perfluoropentane; PSDT, photo-sonodynamic therapy; LPHNPs, lipid-polymer hybrid nanoparticles; TOI_HNPs, folate-targeted LPHNPs-loaded ICG/PFP-carrying oxygen; FITC, fluorescein isothiocyanate.

We also measured the amount of ROS produced by each treatment group in the free system, using SOSG as an indicator. Figure $6 \mathrm{C}$ shows the percentage increase of fluorescence intensity of SOSG after laser and US treatment for degassed PBS, ICG, TO_HNPs, TI_HNPs, OI_PNPs, and TOI_HNPs. After treatment, the SOSG fluorescence intensity revealed a significant increase in TOI_HNPs $(203.87 \% \pm 9.74 \%)$ and OI_PNPs $(189.23 \% \pm 14.05 \%)$ when compared with free ICG $(127.20 \% \pm 2.74 \%)$, TO_HNPs $(77.13 \% \pm 18.40 \%)$, and TI_HNPs $(152.60 \% \pm 7.92 \%)$. Interestingly, there was no significant difference between
TOI_HNPs and OI_PNPs, because the ICG concentration of the two nanoagents was the same in the free system. It is suggested that the laser and US pulses effectively introduced the PFP phase shift, ruptured, and effectively released oxygen. Furthermore, the presence of both oxygen and photosensitizers can be triggered to produce ROS in the cell-free system. ${ }^{4}$

Subsequently, we measured the expression levels of HIF- $1 \alpha$ and IL- 6 protein to explore the mechanism of PSDT. In nonhypoxic conditions, growth factors, cytokines, and other signaling molecules tend to accumulate HIF- $1 \alpha$ protein in cells. ${ }^{46}$ After the SKOV3 cells were exposed to 

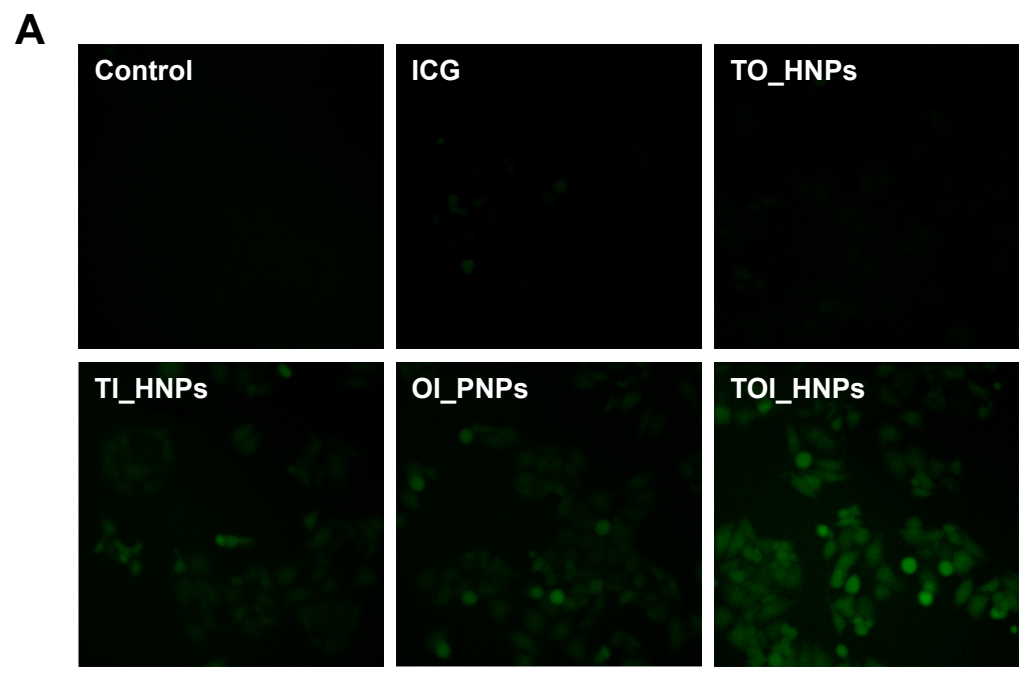

B

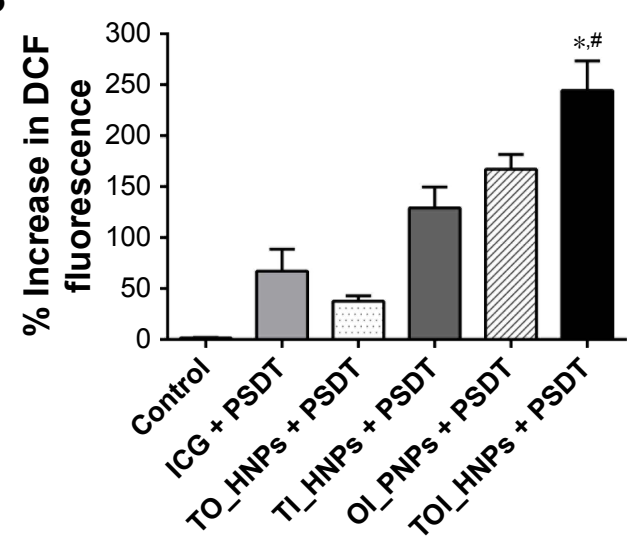

D

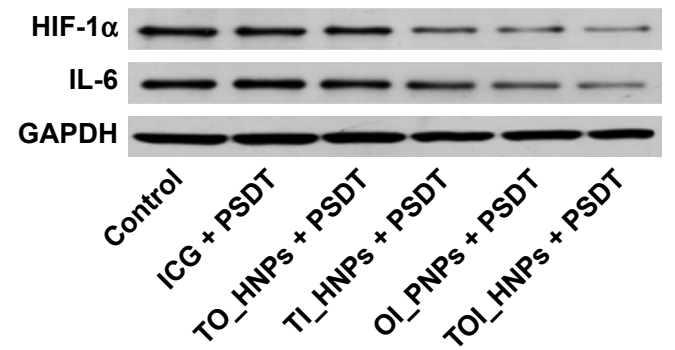

C

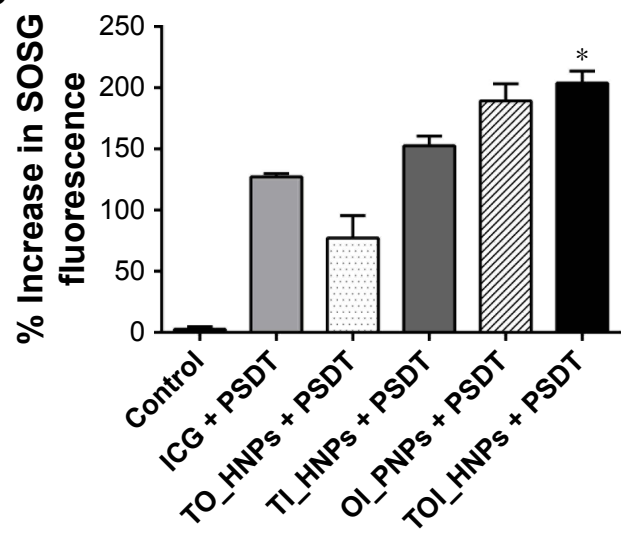

E

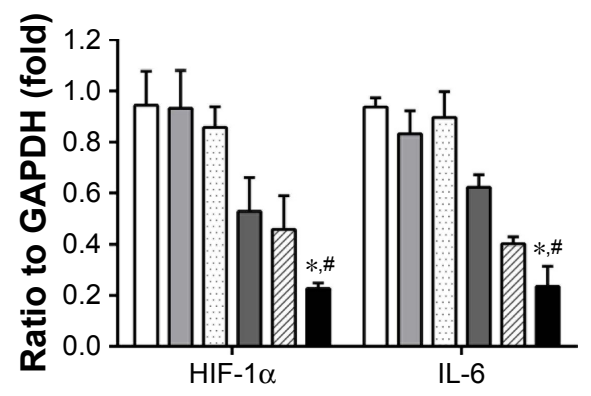

Figure 6 The underlying mechanism of therapy.

Notes: (A) The intracellular ROS production was detected by fluorescence microscopy in SKOV3 cells incubated with DCFH-DA. Scale bar is $50 \mu \mathrm{m}$. (B) Percentage increase in DCF fluorescence for ICG, TO_HNPs, TI_HNPs, OI_PNPs, and TOI_HNPs incubated with SKOV3 cells upon exposure to laser and US measured by fluorescence microplate reader. (C) Percentage increase in SOSG fluorescence for ICG, TO_HNPs, TI_HNPs, OI_PNPs, and TOI_HNPs with laser and US exposure. (D, E) HIF-I $\alpha$ and IL-6 protein expression in SKOV3 cells was analyzed by Western blot after different treatments. GAPDH was used as an internal reference. Compared with control, $* P<0.05$; compared with other groups, ${ }^{\#} P<0.05$.

Abbreviations: DCF, 2',7'-dichlorofluorescein; DCFH-DA, 2',7'-dichlorofluorescin diacetate; HIF-I $\alpha$, hypoxia-inducible factor I-alpha; ICG, indocyanine green; LPHNPs, lipid-polymer hybrid nanoparticles; PFP, perfluoropentane; OI_PNPs, PLGA NPs-encapsulated ICG and PFP-carrying oxygen; SOSG, Singlet Oxygen Sensor Green; TOI_HNPs, folate-targeted LPHNPs-loaded ICG/PFP-carrying oxygen; US, ultrasound; PLGA, poly (lactic-co-glycolic acid); TO_HNPs, folate-targeted LPHNPs-loaded PFPcarrying oxygen; TI_HNPs, folate-targeted LPHNPs-loaded ICG; GAPDH, glyceraldehyde phosphate dehydrogenase. 
different treatments, the expression of HIF-1 $\alpha$ was significantly decreased in the TOI_HNPs + PSDT group, with approximately 0.24 -fold compared with the control group. In addition, the HIF-1 $\alpha$ expression levels of OI_PNPs + PSDT and TI_HNPs + PSDT groups were 0.48 -fold and 0.56 -fold, respectively, which was lower than the untreated group (Figure 6D and E). Some studies showed that the elevated IL-6 and phosphoSTAT3 levels were associated with the development of paclitaxel resistance on ovarian cancer. ${ }^{47}$ Similarly, compared with the control group, the expression of IL-6 was significantly decreased in the TOI_HNPs + PSDT group.

In our study, the TOI_HNPs were assembled with ICG, PFP, and oxygen into lipid-PLGA hybrid nanocarriers. The synthesized NPs have many unique advantages: 1) stability: the stability of hybrid NPs was outstanding than lipid-NPs, exerting long-lasting anti-cancer effect; 2) active targeting ability: cell uptake was achieved by active targeting rather than passive internalization, after coating with FA-lipid membrane on the surface of PLGA NPs; and 3) triggered activity: under laser irradiation, the core-shell NPs can be transformed into microns. The microbubbles were ruptured to effectively release ICG and oxygen when exposed to US. In other words, TOI_HNPs can be stimulated, combining laser with US, to exerted series reactions. Our results demonstrated that TOI_HNPs-mediated PSDT can produce a large number of ROS (including singlet oxygen, superoxide anions, and hydroxyl radicals), which mediated oxidative stress and induced indiscriminately damage of lipids, proteins, and DNA, leading to cancer cell apoptosis. ${ }^{48,49}$ At the same time, the destruction of TOI_HNPs can release oxygen, altering the oxygen environment of SKOV3 cells and thus downregulating HIF-1 $\alpha$ expression. ${ }^{46}$ Finally, we found that TOI_HNPs-mediated PSDT treatment of ovarian cancer also downregulated the expression of IL-6, indicating that PSDT may change the biological behavior of ovarian cancer by affecting the IL-6/stat-3 pathway, which was conducive to overcome drug resistance..$^{47,50}$

\section{Limitations}

The experimental work described in this paper still has several limitations. First, we unexpectedly found that TOI_ HNPs-mediated PSDT may alter the IL-6/stat-3 signaling pathway, and evaluating the ability of reverse drug resistance need to be explored by encapsulating chemotherapeutic drugs into NPs or establishing drug resistance models. Second, our experiments showed that PSDT-mediated destruction of oxygen and ICG-loaded phase-transition NPs can produce synergistic effects on SKOV3 cells in vitro. In the following work, we will further investigate the targeting ability and therapeutic effects in vivo.

\section{Conclusion}

We have successfully constructed core-shell hybrid nanosystem as TOI_HNPs, which are more stable than lipid NPs and have high encapsulation efficiency and active targeting ability than PLGA_NPs. Importantly, our study demonstrated that laser and US activation of TOI_HNPs could be used as a dual-mode imaging guidance with synergistic therapeutic effects. We believe this kind of hybrid NPs may provide a new strategy for image-guided cancer therapy without the need for conventional chemotherapy.

\section{Acknowledgments}

The authors are grateful to Dr Ronald X. Xu (Department of Precision Machinery and Precision Instrumentation, University of Science and Technology of China, Hefei, Anhui, China) for technical advice, Dr Pan Li and Lan Hao (Institute of Ultrasound Imaging, Second Hospital of Chongqing Medical University, and Chongqing, China) for technical advice on dual-mode imaging, and Dr Tinghe Yu (Director of Key Medical laboratory of Obstetrics and Gynecology, The Second Affiliated Hospital, Chongqing Medical University, Chongqing, China) for the generous support of the experimental facilities. This study was supported by the Natural Science Foundation of China (81572558, 81630047, 31630026) and Natural Science Foundation of Chongqing (cstc2018jcyjAX0103, cstc2018jcyjAX0223).

\section{Author contributions}

All authors contributed to data analysis, drafting or revising the article, gave final approval of the version to be published, and agree to be accountable for all aspects of the work.

\section{Disclosure}

The authors report no conflicts of interest in this work.

\section{References}

1. Torre LA, Bray F, Siegel RL, Ferlay J, Lortet-Tieulent J, Jemal A. Global cancer statistics, 2012. CA Cancer J Clin. 2015;65(2):87-108.

2. Lin H, Chen Y, Shi J. Nanoparticle-triggered in situ catalytic chemical reactions for tumour-specific therapy. Chem Soc Rev. 2018;47(6): 1938-1958.

3. Wicki A, Witzigmann D, Balasubramanian V, Huwyler J. Nanomedicine in cancer therapy: challenges, opportunities, and clinical applications. J Control Release. 2015;200:138-157.

4. Qian X, Zheng Y, Chen Y. Micro/nanoparticle-augmented sonodynamic therapy (SDT): breaking the depth shallow of photoactivation. Adv Mater. 2016;28(37):8097-8129.

5. Shi J, Kantoff PW, Wooster R, Farokhzad OC. Cancer nanomedicine: progress, challenges and opportunities. Nat Rev Cancer. 2017;17(1):20-37. 
6. Bobo D, Robinson KJ, Islam J, Thurecht KJ, Corrie SR. Nanoparticlebased medicines: a review of FDA-approved materials and clinical trials to date. Pharm Res. 2016;33(10):2373-2387.

7. Tang Q, Cui J, Tian Z, et al. Oxygen and indocyanine green loaded phase-transition nanoparticle-mediated photo-sonodynamic cytotoxic effects on rheumatoid arthritis fibroblast-like synoviocytes. Int $J$ Nanomedicine. 2017;12:381-393.

8. Zheng M, Zhao P, Luo Z, et al. Robust ICG theranostic nanoparticles for folate targeted cancer imaging and highly effective photothermal therapy. ACS Appl Mater Interfaces. 2014;6(9):6709-6716.

9. Wu P, Zhao T, Wang S, Hou X. Semiconductor quantum dots-based metal ion probes. Nanoscale. 2014;6(1):43-64.

10. Abrahamse H, Hamblin MR. New photosensitizers for photodynamic therapy. Biochem J. 2016;473(4):347-364.

11. Hadinoto K, Sundaresan A, Cheow WS. Lipid-polymer hybrid nanoparticles as a new generation therapeutic delivery platform: a review. Eur J Pharm Biopharm. 2013;85(3 Pt A):427-443.

12. Mandal B, Bhattacharjee H, Mittal N, et al. Core-shell-type lipid-polymer hybrid nanoparticles as a drug delivery platform. Nanomedicine. 2013;9(4):474-491.

13. Kamaly N, Yameen B, Wu J, Farokhzad OC. Degradable controlledrelease polymers and polymeric nanoparticles: mechanisms of controlling drug release. Chem Rev. 2016;116(4):2602-2663.

14. Sercombe L, Veerati T, Moheimani F, Wu SY, Sood AK, Hua S. Advances and challenges of liposome assisted drug delivery. Front Pharmacol. 2015;6:286.

15. Giraudeau C, Moussaron A, Stallivieri A, Mordon S, Frochot C. Indocyanine green: photosensitizer or chromophore? Still a debate. Curr Med Chem. 2014;21(16):1871-1897.

16. Lu N, Fan W, Yi X, et al. Biodegradable hollow mesoporous organosilica nanotheranostics for mild hyperthermia-induced bubbleenhanced oxygen-sensitized radiotherapy. ACS Nano. 2018;12(2): $1580-1591$.

17. Jian J, Liu C, Gong Y, et al. India ink incorporated multifunctional phase-transition nanodroplets for photoacoustic/ultrasound dualmodality imaging and photoacoustic effect based tumor therapy. Theranostics. 2014;4(10):1026-1038.

18. Xu J, Zhou J, Zhong Y, et al. Phase transition nanoparticles as multimodality contrast agents for the detection of thrombi and for targeting thrombolysis: in vitro and in vivo experiments. ACS Appl Mater Interfaces. 2017;9(49):42525-42535.

19. Zhou Y, Wang Z, Chen Y, et al. Microbubbles from gas-generating perfluorohexane nanoemulsions for targeted temperature-sensitive ultrasonography and synergistic HIFU ablation of tumors. Adv Mater. 2013;25(30):4123-4130.

20. Ledermann JA, Canevari S, Thigpen T. Targeting the folate receptor: diagnostic and therapeutic approaches to personalize cancer treatments. Ann Oncol. 2015;26(10):2034-2043.

21. Piao JG, Wang L, Gao F, You YZ, Xiong Y, Yang L. Erythrocyte membrane is an alternative coating to polyethylene glycol for prolonging the circulation lifetime of gold nanocages for photothermal therapy. ACS Nano. 2014;8(10):10414-10425.

22. Zhang L, Chan JM, Gu FX, et al. Self-assembled lipid-polymer hybrid nanoparticles: a robust drug delivery platform. ACS Nano. 2008; 2(8):1696-1702.

23. Zheng $\mathrm{C}$, Zheng $\mathrm{M}$, Gong $\mathrm{P}$, et al. Indocyanine green-loaded biodegradable tumor targeting nanoprobes for in vitro and in vivo imaging. Biomaterials. 2012;33(22):5603-5609.

24. Bose RJ, Arai Y, Ahn JC, Park H, Lee SH. Influence of cationic lipid concentration on properties of lipid-polymer hybrid nanospheres for gene delivery. Int J Nanomedicine. 2015;10:5367-5382.

25. Pisani E, Fattal E, Paris J, Ringard C, Rosilio V, Tsapis N. Surfactant dependent morphology of polymeric capsules of perfluorooctyl bromide: influence of polymer adsorption at the dichloromethane-water interface. J Colloid Interface Sci. 2008;326(1):66-71.
26. Zhang L, Wang D, Yang K, et al. Mitochondria-targeted artificial "nanoRBCs" for amplified synergistic cancer phototherapy by a single NIR irradiation. Adv Sci. 2018;5(8):1800049.

27. Bhattacharjee S. DLS and zeta potential - what they are and what they are not? J Control Release. 2016;235:337-351.

28. Sheng D, Liu T, Deng L, et al. Perfluorooctyl bromide \& indocyanine green co-loaded nanoliposomes for enhanced multimodal imagingguided phototherapy. Biomaterials. 2018;165:1-13.

29. Ramazani F, Chen W, van Nostrum CF, et al. Strategies for encapsulation of small hydrophilic and amphiphilic drugs in PLGA microspheres: state-of-the-art and challenges. Int J Pharm. 2016;499(1-2):358-367.

30. Iqbal M, Zafar N, Fessi H, Elaissari A. Double emulsion solvent evaporation techniques used for drug encapsulation. Int $J$ Pharm. 2015;496(2):173-190.

31. Giesecke T, Hynynen K. Ultrasound-mediated cavitation thresholds of liquid perfluorocarbon droplets in vitro. Ultrasound Med Biol. 2003; 29(9):1359-1365.

32. Han L, Xiong P, Bai J, Che S. Spontaneous formation and characterization of silica mesoporous crystal spheres with reverse multiply twinned polyhedral hollows. J Am Chem Soc. 2011;133(16):6106-6109.

33. Chen Q, Yu J, Kim K. Review: optically-triggered phase-transition droplets for photoacoustic imaging. Biomed Eng Lett. 2018;8(2):223-229.

34. Manshian BB, Moyano DF, Corthout N, et al. High-content imaging and gene expression analysis to study cell-nanomaterial interactions: the effect of surface hydrophobicity. Biomaterials. 2014;35(37):9941-9950.

35. Kim IY, Joachim E, Choi H, Kim K. Toxicity of silica nanoparticles depends on size, dose, and cell type. Nanomedicine. 2015;11(6): $1407-1416$.

36. Soenen SJ, Illyes E, Vercauteren D, et al. The role of nanoparticle concentration-dependent induction of cellular stress in the internalization of non-toxic cationic magnetoliposomes. Biomaterials. 2009;30(36):6803-6813.

37. Li L, Fernández-Cruz ML, Connolly M, et al. The potentiation effect makes the difference: non-toxic concentrations of $\mathrm{ZnO}$ nanoparticles enhance $\mathrm{Cu}$ nanoparticle toxicity in vitro. Sci Total Environ. 2015;505:253-260.

38. Bakhshizadeh M, Moshirian T, Esmaily H, Rajabi O, Nassirli H, Sazgarnia A. Sonophotodynamic therapy mediated by liposomal zinc phthalocyanine in a colon carcinoma tumor model: role of irradiating arrangement. Iran J Basic Med Sci. 2017;20(10):1088-1092.

39. Xu F, Hu M, Liu C, Choi SK. Yolk-structured multifunctional upconversion nanoparticles for synergistic photodynamic-sonodynamic antibacterial resistance therapy. Biomater Sci. 2017;5(4):678-685.

40. Wang P, Li C, Wang X, et al. Anti-metastatic and pro-apoptotic effects elicited by combination photodynamic therapy with sonodynamic therapy on breast cancer both in vitro and in vivo. Ultrason Sonochem . 2015;23:116-127.

41. Wang H, Wang X, Wang P, Zhang K, Yang S, Liu Q. Ultrasound enhances the efficacy of chlorin E6-mediated photodynamic therapy in MDA-MB-231 cells. Ultrasound Med Biol. 2013;39(9):1713-1724.

42. Liu Y, Wang P, Liu Q, Wang X. Sinoporphyrin sodium triggered sono-photodynamic effects on breast cancer both in vitro and in vivo. Ultrason Sonochem. 2016;31:437-448.

43. Li Q, Liu Q, Wang P, Feng X, Wang H, Wang X. The effects of Ce6-mediated sono-photodynamic therapy on cell migration, apoptosis and autophagy in mouse mammary 4T1 cell line. Ultrasonics. 2014;54(4):981-989.

44. Ma R, Wu Q, Si T, Chang S, Xu RX, Rx X. Oxygen and indocyanine green loaded microparticles for dual-mode imaging and sonodynamic treatment of cancer cells. Ultrason Sonochem. 2017;39:197-207.

45. Nomikou N, Curtis K, Mcewan C, et al. A versatile, stimulus-responsive nanoparticle-based platform for use in both sonodynamic and photodynamic cancer therapy. Acta Biomater. 2017;49:414-421.

46. Masoud GN, Li W. HIF-1 $\alpha$ pathway: role, regulation and intervention for cancer therapy. Acta Pharm Sin B. 2015;5(5):378-389. 
47. Yanaihara N, Hirata Y, Yamaguchi N, et al. Antitumor effects of interleukin-6 (IL-6)/interleukin-6 receptor (IL-6R) signaling pathway inhibition in clear cell carcinoma of the ovary. Mol Carcinog. 2016;55(5):832-841.

48. Diebold L, Chandel NS. Mitochondrial ROS regulation of proliferating cells. Free Radic Biol Med. 2016;100:86-93.

49. Moloney JN, Cotter TG. ROS signalling in the biology of cancer. Semin Cell Dev Biol. 2018;80:50-64.
50. Suh YA, Jo SY, Lee HY, Lee C. Inhibition of IL-6/STAT3 axis and targeting Axl and Tyro3 receptor tyrosine kinases by apigenin circumvent taxol resistance in ovarian cancer cells. Int $J$ Oncol. 2015;46(3):1405-1411.

International Journal of Nanomedicine

\section{Publish your work in this journal}

The International Journal of Nanomedicine is an international, peerreviewed journal focusing on the application of nanotechnology in diagnostics, therapeutics, and drug delivery systems throughout the biomedical field. This journal is indexed on PubMed Central, MedLine, CAS, SciSearch $®$, Current Contents $® /$ Clinical Medicine,
Journal Citation Reports/Science Edition, EMBase, Scopus and the Elsevier Bibliographic databases. The manuscript management system is completely online and includes a very quick and fair peer-review system, which is all easy to use. Visit http://www.dovepress.com/ testimonials.php to read real quotes from published authors.

Submit your manuscript here: http://www.dovepress.com/international-journal-of-nanomedicine-journal 